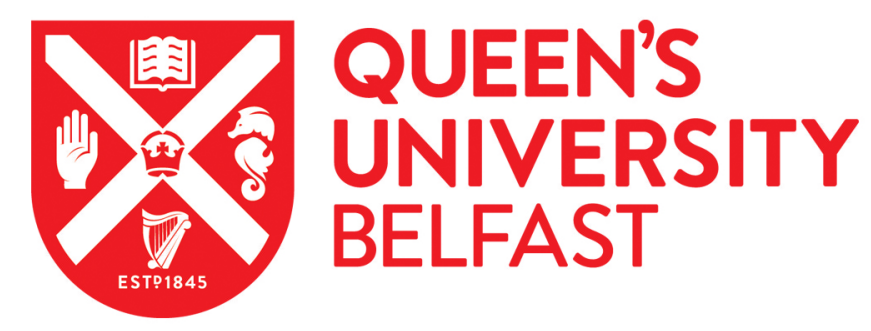

\title{
Effective end wall profiling rules for a highly loaded compressor cascade
}

Li, X., Chu, W., Wu, Y., Zhang, H., \& Spence, S. (2016). Effective end wall profiling rules for a highly loaded compressor cascade. Proceedings of the Institution of Mechanical Engineers, Part A: Journal of Power and Energy. https://doi.org/10.1177/0957650916649084

Published in:

Proceedings of the Institution of Mechanical Engineers, Part A: Journal of Power and Energy

Document Version:

Peer reviewed version

Queen's University Belfast - Research Portal:

Link to publication record in Queen's University Belfast Research Portal

Publisher rights

Copyright (C 2016 by Institution of Mechanical Engineers

\section{General rights}

Copyright for the publications made accessible via the Queen's University Belfast Research Portal is retained by the author(s) and / or other copyright owners and it is a condition of accessing these publications that users recognise and abide by the legal requirements associated with these rights.

Take down policy

The Research Portal is Queen's institutional repository that provides access to Queen's research output. Every effort has been made to ensure that content in the Research Portal does not infringe any person's rights, or applicable UK laws. If you discover content in the Research Portal that you believe breaches copyright or violates any law, please contact openaccess@qub.ac.uk. 


\section{Effective End Wall Profiling Rules for a Highly Loaded Compressor Cascade LI Xiangjun' ${ }^{1}$, CHU Wulii,2*, WU Yanhui ${ }^{1}$, ZHANG Haoguang1, Stephen Spence ${ }^{3}$ \\ 1. School of Power and Energy, Northwestern Polytechnical University, Xi'an 710129, PR China \\ 2. Collaborative Innovation Center of Advanced Aero-Engine, Beijing 100191, PR China \\ 3. School of Mechanical and Aerospace Engineering, Queen's University Belfast, Belfast BT9 5AH, UK}

\section{Abstract}

This paper presents a numerical study of a linear compressor cascade to investigate the effective end wall profiling rules for highly-loaded axial compressors. The first step in the research applies a correlation analysis for the different flow field parameters by a data mining over 600 profiling samples to quantify how variations of loss, secondary flow and passage vortex interact with each other under the influence of a profiled end wall. The result identifies the dominant role of corner separation for control of total pressure loss, providing a principle that only in the flow field with serious corner separation does the does the profiled end wall change total pressure loss, secondary flow and passage vortex in the same direction. Then in the second step, a multi-objective optimization of a profiled end wall is performed to reduce loss at design point and near stall point. The development of effective end wall profiling rules is based on the manner of secondary flow control rather than the geometry features of the end wall. Using the optimum end wall cases from the Pareto front, a quantitative tool for analyzing secondary flow control is employed. The driving force induced by a profiled end wall on different regions of end wall flow are subjected to a detailed analysis and identified for their positive/negative influences in relieving corner separation, from which the effective profiling rules are further confirmed. It is found that the profiling rules on a cascade show distinct differences at design point and near stall point, thus loss control of different operating points is generally independent.

\section{Keywords}

Compressor, profiled end wall, cascade, corner separation, numerical simulation

\section{Introduction}

Nowadays compressors are commonly designed with higher aerodynamic load to meet the demand of thrust to weight ratio of a modern aeroengine. Driven by the high pressure gradient across the blade passage, the cross-passage flow within the end wall region is intensified and therefore accumulates more of boundary layer fluid into the suction side (SS) corner ${ }^{[1]}$. As a result, this commonly leads to significant three-dimensional corner separation and increase in the total pressure loss.

During the past decades, numerous methods have been put forward to control the end wall flow of turbomachines. Next to the possibility of using three-dimensional blade shaping, boundary layer suction/blowing and vortex generators, non-axisymmetric profiled end wall (PEW, also known as end wall contouring) is a good additional option to improve the performance of compressors. As a promising passive flow control measure, PEW was initially proposed in turbine cascade ${ }^{[2]}$. Research into PEW can be broadly separated into two categories by the approach of how the end wall geometry is designed. One is the well known CFD based numerical optimization, which is widely used in both turbines and compressors; this is very time consuming, but generally produces satisfactory performance. The end wall geometry is commonly parameterized and controlled using a set of free varying points. An optimization procedure is then performed via a certain algorithm to get the optimum result. The other method is based on previous experience, which is well developed in turbines but seldom published in compressors except for Hu's paper ${ }^{[3]}$. The perturbation of the end wall geometry in this approach is defined using some smooth function, which is commonly controlled by very few parameters. The values of parameters are typically given by previous experience and adjusted by trial-and-error to find a satisfactory result.

The basic principle of PEW is to employ concave/convex contours on the end wall surface to change the static pressure. Studies in turbines show that a non-axisymmetric end wall with an upslope surface from SS to PS is commonly beneficial to the compressor performance because it reduces the cross-passage pressure difference and the secondary loss. The "upslope rule" thus becomes a design guideline and is employed in the so-called conventional contouring approach for performance improvement of turbines ${ }^{[17]}$.

However, in compressor applications, this profiling rule is supported by only a few studies. The empirically based study of $\mathrm{Hu}$, as previously mentioned, employed this rule on the hub end wall of a low-load rotor and improved the efficiency by $0.45 \%$. Wu ${ }^{[4]}$ conducted an optimization on the hub end wall of a highly-loaded axial flow compressor cascade. The result showed a large concave near PS with

\footnotetext{
${ }^{*}$ Corresponding author: CHU Wuli, School of power and energy, Northwestern polytechnical university, Dongda Road, Chang 'an district, Xi'an, PR China.E-mail: wlchu@nwpu.edu.cn.
} 
a slight convex near SS, just as described by the upslope rule. Additionally, the optimization studies of Liu ${ }^{[5]}$, Zhao ${ }^{[6]}$ and Reutter ${ }^{[7]}$ all gave similar results with some minor differences, where the cross-passage flow was suppressed in the end wall region and finally obtained significant loss reduction $(2 \%-5 \%$ of the total) or efficiency improvement (over $0.3 \%$ ).

There were also studies on PEW employing the numerical optimization but shows different results.

Harvey researched a linear cascade ${ }^{[8]}$ and obtained profiling with a convex near SS and a concave in mid-pitch region. The cross flow was seen to intensify in the rear part of the passage and the overall total pressure loss was reduced by about 7\%. In a further study on the third stage of a 6-stage HP compressor ${ }^{[9]}$, the optimized non-axisymmetric end wall possessed a concave in the front region near the SS followed by a convex in the rear region. The loss core of the suction leg of the horse shoe vortex moved off the end wall and on to the suction surface of the aerofoil, finally reducing the total pressure loss coefficient by 0.02 .

In Reising's ${ }^{[10,11]}$ study, multi-objective PEW optimization was applied to a compressor stage and produced an opposite result to the upslope rule. The optimum end wall geometries on hub and casing end wall of the stator sank near the PS and rose in mid-pitch, with a different shape near the SS; however, but both end walls suppressed the development of corner separation by accelerating the cross-passage secondary flow in the rear passage. The optimized rotor hub also possessed a similar shaping. The cross pressure gradient did increase in value, but turned less perpendicular to the streamlines, which finally suppressed secondary flow and increased efficiency.

Additionally, some more optimization exercises obtained end wall geometries that partially agreed with the upslope rule. This can be seen in Lepot's investigation ${ }^{[12]}$, where the end wall was profiled together with the blade and obtained a combined PEW which agreed with the upslope rule in the front part of the passage while appearing contrary to it in the rest region. In Li's ${ }^{[13]}$ research, the optimized PEW was found to slow down cross-passage flow in the mid-pitch region, accelerate it when approaching the SS and then slow it down again in the region of the SS corner. A recent study of Mahesh $^{[14]}$ conducted an optimization profiling employing 130 control points on the hub end wall surface. The optimized end wall geometry presented a local convex surface in the mid-chord of the SS corner and a concave surface in the mid-chord of the PS corner, with large amount of convex and concaves spreading in the wide mid-pitch region of the end wall, thus making a very complex profile to analyze.

The above shows that research on optimization of PEW did not even reach a common view about the effective end wall profiling rules. This inconsistency may likely come from the complexity of the diffusing flow in compressors. It is known that the development of corner separation can be sensitive to local pressure gradient, which is easily altered by a slight change of the PEW geometry or even the same geometry with a different inflow angle. The upslope rule only provides a superficial guidance for the end wall geometry; although it has a physics basis, it is not actually effective for end wall profiling in compressor. This may also explain why the time-saving PEW approach, employing empirical experience and trial-and-error, is so much underdeveloped and seldom published in compressors.

To make end wall profiling in compressors more effective and widely used, the empirical design approach of PEW should be given greater consideration as in turbines. More effective profiling rules should be developed focusing on the control of secondary flow provided by PEW rather than its geometry feature. The following two aspects are critical:

i. clarify the interaction among different flow structures (vortex, secondary flow and separation) and their correlation with loss for general profiling principles;

ii. Identify the effective and specific method by which end wall shaping controls the above flow structures and loss generation.

After a brief introduction of the objective in study and the numerical setup in section2, the former problem will be discussed in section 3 with the help of data mining. The latter will be discussed in section 4, based on the conclusion of the correlation analysis and the results of a multi-objective optimization design of PEW.

\section{Compressor cascade and CFD method}

A linear compressor cascade with an inlet boundary layer is chosen for this study. The reason is partly because it has already been experimentally studied in detail, but more importantly because it is uniform in the span-wise direction and has no leakage flow and boundary layer skew, thus eliminating the co-influences of some complex factors in real compressors. Since there is no centrifugal force and Coriolis force, studies in this cascade are actually indicative of an ideal condition in the tip region of the stator where there is no influence of different span-wise load as well as non-uniform inlet incidence, except for an inlet boundary layer and cross-passage secondary flow. 


\subsection{Compressor cascade in study}

The cascade in study is a typical highly-loaded low-speed linear compressor cascade, and has been numerically and experimentally studied by Zhang ${ }^{[15]}$. Fig.1 provides its overview. The circular-arc element is designed with a $50^{\circ}$ flow turning angle and its diffusion factor reaches 0.5 . According to Zhang's study ${ }^{[15,16]}$, corner separation in this cascade occupies a large area over the suction surface rather than being confined to the hub-SS corner, mainly because of the strong pitchwise pressure gradient. Table 1 gives the main design parameters of the cascade. More details about the test rig can be found in reference [15].

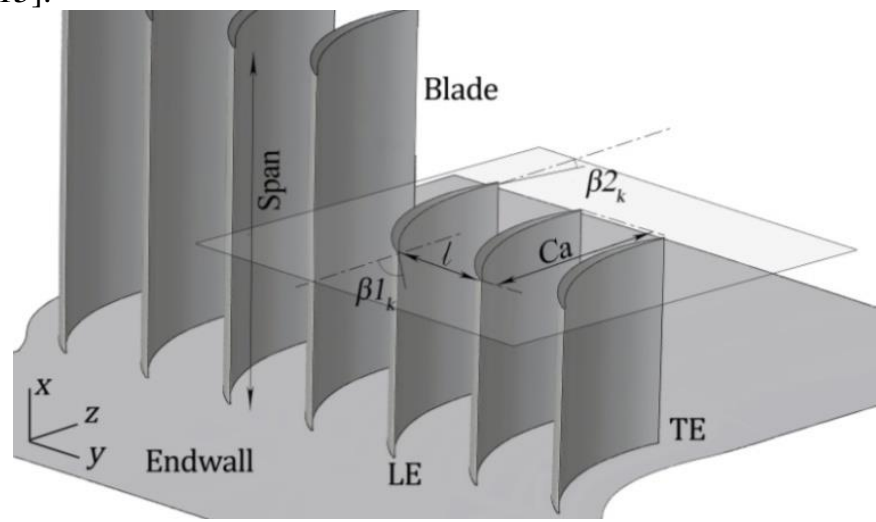

Fig.1 Overview of cascade

Table 1 Design parameters of cascade ${ }^{[16]}$

\begin{tabular}{ll}
\hline parameters & value \\
\hline Height of blade, span & $0.2 \mathrm{~m}$ \\
axial chord, $\mathrm{Ca}$ & $0.107 \mathrm{~m}$ \\
solidity, $\mathrm{C} / \mathrm{s}$ & 2.14 \\
inlet metal angle, $\beta 1_{k}$ & $52^{\circ}$ \\
outlet metal angle, $\beta 2_{k}$ & $12^{\circ}$ \\
stagger angle & $20.5^{\circ}$ \\
\hline
\end{tabular}

\subsection{CFD method}

Figure 2 provides an over view of the grids. They are generated using AutoGrid5. Only a half-span passage is generated with a mirror boundary at $50 \%$ span because of its span-wise symmetrical structure. The inlet and outlet passages both use long extended "H" blocks. The former extends to $1.2 \mathrm{Ca}$ upstream of the leading edge (LE), which is the location where experimental measurements were taken. The latter reaches 2.0 Ca downstream of the trailing edge (TE) to ensure an adequate mixing of the outlet flow. The blade-to-blade flow surface employs refined nodes in the center with "O4H" topology to achieve a higher degree of orthogonality. The numbers of nodes in some important sections are also indicated in Fig.2. The above mentioned details of the numerical model are the same as those reported in previous work ${ }^{[13]}$, in which the grid development was validated by experimental measurements after going through a grid convergence study. In this paper, the grid distribution is changed only on the span-wise direction. The number of span-wise nodes has been doubled from the previous study to a value of 81 , and in particular has been refine in the hub region to better predict the end wall flow. As the result, the overall number of nodes is twice as before and reaches around 1,538, 000. The average $\mathrm{y}^{+}$of first grid layer is less than 1 .

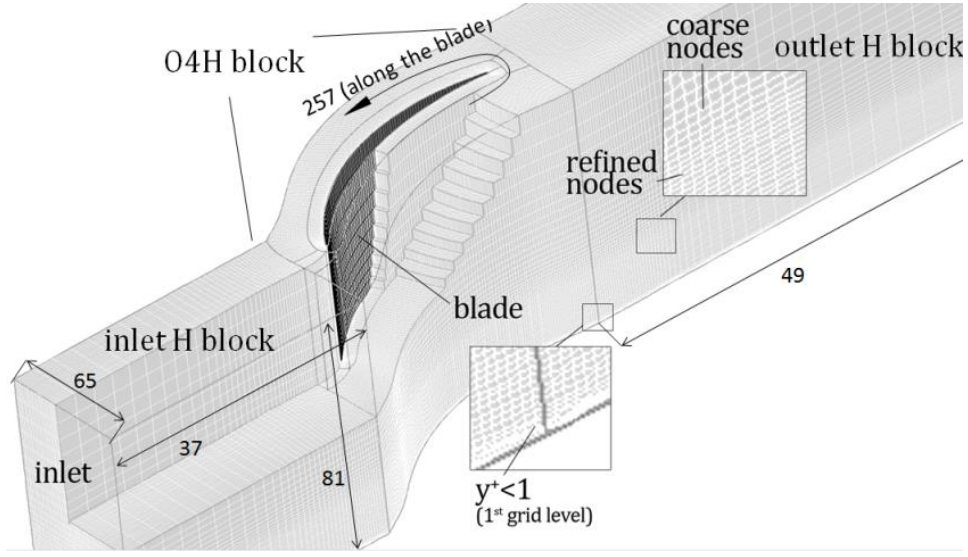

Fig. 2 Overview of grids 
At inlet, incidence was set uniformly along the span and the velocity profile was specified according to the experimental data, as shown in Fig.3. The thickness of the end wall boundary layer reaches about $12.5 \%$ span. At the outlet boundary, static pressure was set to atmospheric pressure. Two operating points were selected here: the design point $\left(\mathrm{DP}\right.$, incidence $=-1^{\circ}$ ) and the near corner-stall point $(\mathrm{NS}$, incidence $=+7^{\circ}$ ) to evaluate the performance of the cascade under different inlet conditions. The simulation was conducted using the commercial code EURANUS. The flow field of the cascade was calculated by solving the steady RANS equations with the two equation k- $\omega$ Wilcox turbulence model. The spatial discrezitation used Jameson's 2nd order cell-centered explicit finite volume scheme and the time stepping used the 4-step Runge-Kutta scheme. It should be noted that at the last operating condition of the compressor cascade, i.e. near the stall point, strong perturbations are often found in the real flow field and leads to instability in the numerical model. However, in this case, the convergence history showed no significant perturbation, even near the stall point. Consequently, the steady simulation was still appropriate for the performance evaluation at the near stall condition. Multi-grids and local time stepping techniques were applied to speed-up convergence.

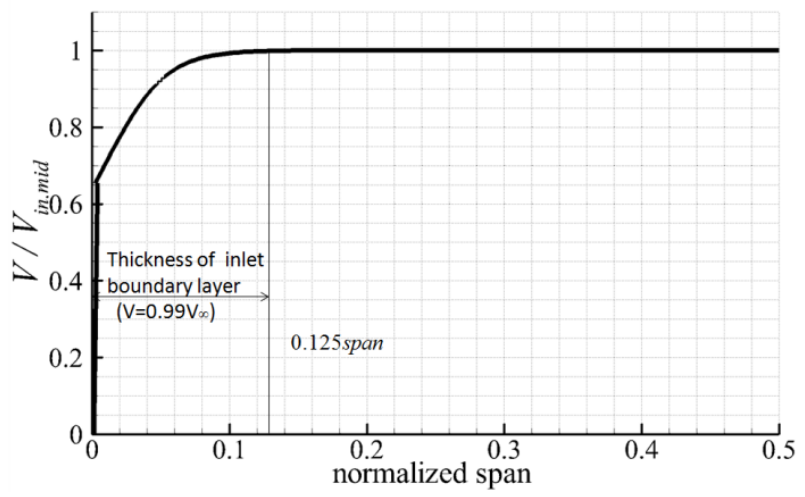

Fig.3 inlet velocity profile

\subsection{Validation of the CFD method}

Figure 4 provides a comparison between the numerical and experimental results of the pitch-wise averaged total pressure loss and flow angle taken $0.4 C a$ downstream of the TE. The Curve labeled "b1" give the result at design point. The simulation shows an over estimation of loss over the full range, however, good agreement is demonstrated with the characteristic shape of the experimental profiles, especially the "S" shape of the loss profile within the first $10 \%$ of span and the location of the under-turning peak in the distribution of the flow angle. This indicates that the coupling effect of the corner separation and the hub cross flow is well predicted. Additionally, another group of data with a thicker inlet boundary layer (thickness reaching 22.5\% span), labeled with "b2", are given for a further comparison. The curves of $\mathrm{b} 1$ and $\mathrm{b} 2$ show that the CFD model calculated the effect of a thicker boundary layer very well - both in magnitude and position of the changes in loss and flow angle, regardless of the absolute difference between the experimental and simulated values. This indicates a satisfactory level of simulation accuracy to calculate the extension of separation.
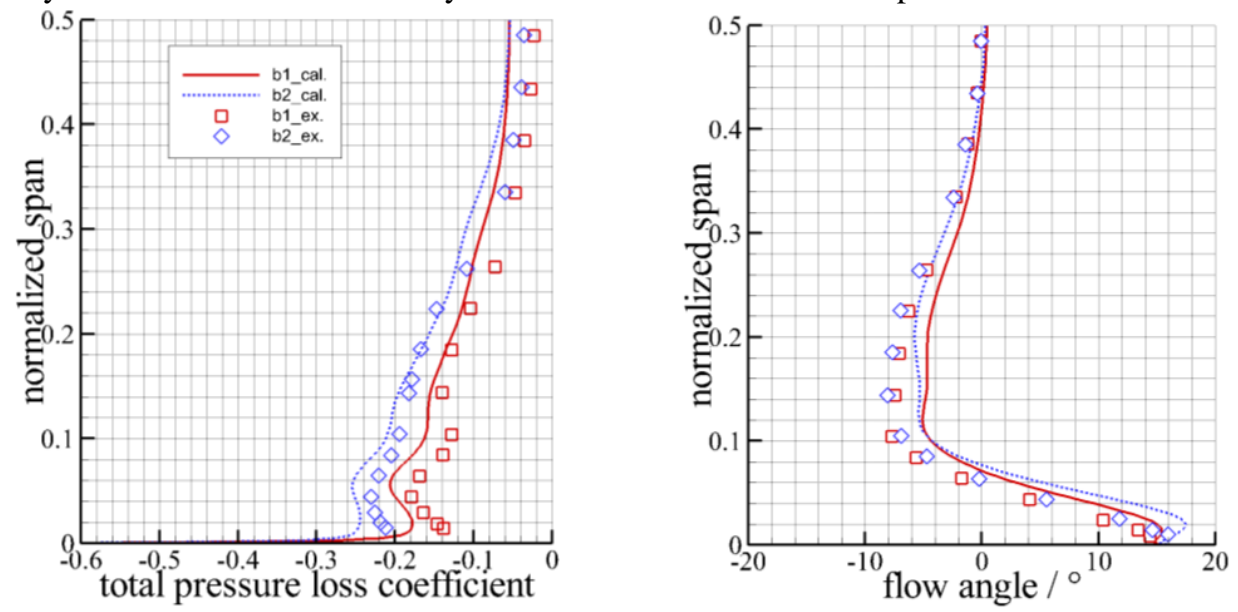

Fig.4 experimental and calculated pitch-average total pressure loss and flow angle at $0.4 \mathrm{Ca}$ downstream of TE

Figure 5 shows the streamline pattern near the SS and hub at design point to judge the accuracy of the CFD method in predicting the corner separation. Near the end wall, the calculated limiting 
streamlines show a strong cross trend and match the result of oil-flow very well. The calculated start point of corner separation, i.e. the saddle point labeled $\mathrm{N}_{\mathrm{N} 2}$, is almost at the same position as the experiment, with a slight discrepancy being apparent in the separation area alongside the SS corner. On the SS, a separation line is seen around 10\%span, indicating the low energy fluid accumulated on the SS. The direction of climb and reversed flow below 10\%span at the rear SS corner agrees with the experiment. However, limited by the CFD method, the separation bubble within $0.2-0.5 \mathrm{Ca}$ is not predicted because the full turbulent assumption makes transition beyond the ability of this simulation.

The separation bubble on the SS can be important for a two-dimensional separation (in quasi-S1 stream surface). The absence of the separation bubble means that the two-dimensional separation starts earlier at the TE. The three-dimensional separation also occupies larger area. This may explains the full-span over estimation of the calculated total pressure loss and flow angle, as shown in Fig.4. Nevertheless, it was judged that the fully turbulent modeling assumption would not significantly influence this study, because the direction of the hub corner flow, which is believed most critical for the development of corner separation, is well predicted. This may explain why CFD shows some discrepancy with the experimental result, while still exhibiting a similar tends and an accurate result for the variation of loss and flow angle at different extents of separation. The deviation between the absolute level of values predicted by CFD and experiments cancels out when comparing between the predicted sets of results for different cases. It was considered that the CFD model had adequate accuracy for the present study.
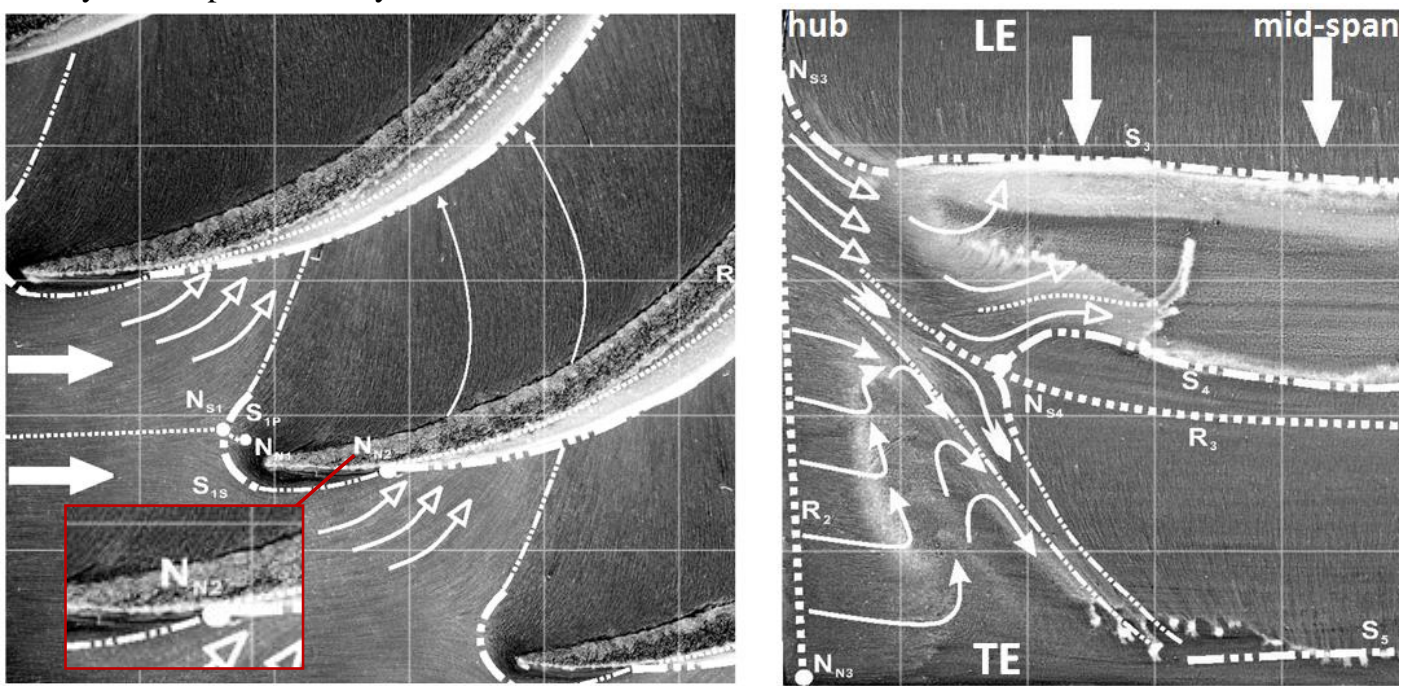

a. experiment result of oil-flow visualization ${ }^{[15]}$
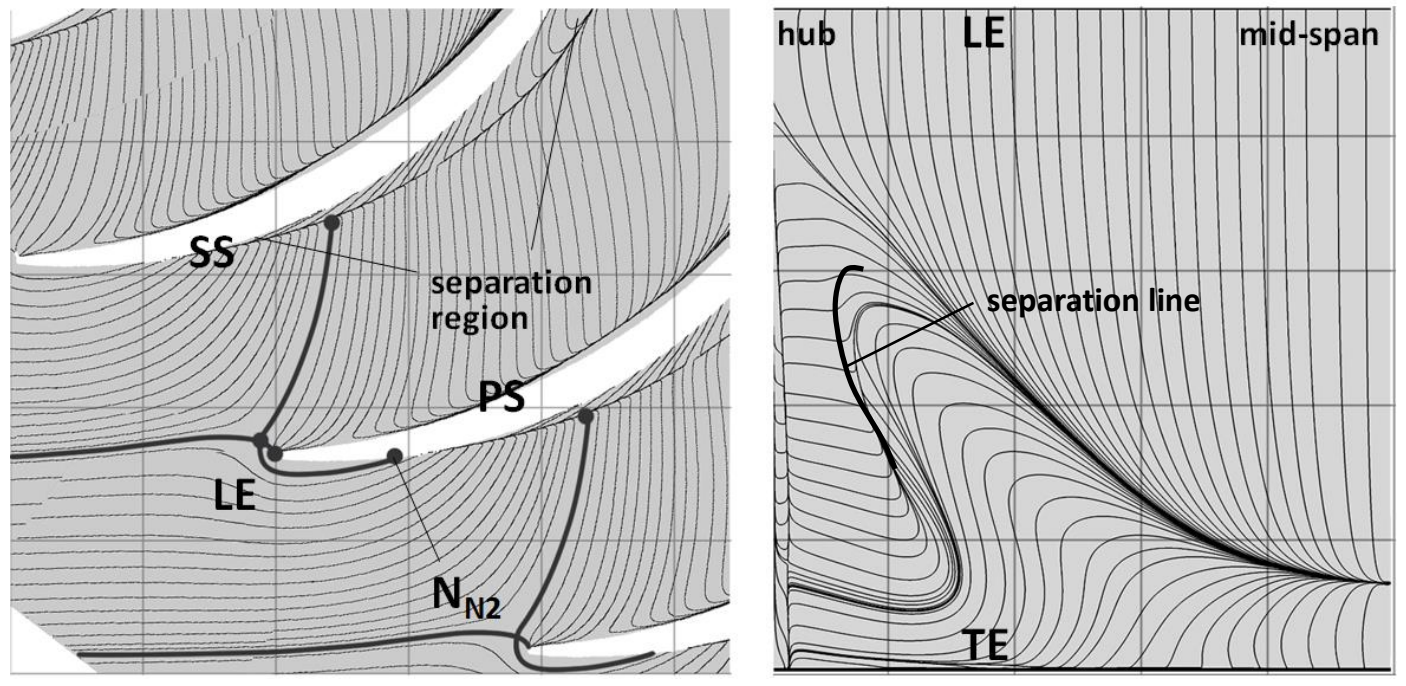

b. calculated limiting streamlines

Fig.5 flow pattern near hub end wall (left) and SS(right)

\section{Correlation analysis for flow structures and loss}

To provide general principles and guidance for end wall design, this section described the application 
of a data mining method on the PEW to analyze the correlation among different flow structures and loss during profiling. The profiling method and the theoretical basis will be presented in subsection 3.1 and 3.2. The coordinate system for all the equations below is illustrated in Fig.1, in which $x, y$ and $z$ are respectively defined as the span-wise, pitchwise and streamwise (axial) direction.

\subsection{Contouring method}

Figure 6 shows the profiling method. The end wall surface was a lofted surface over six Bezier curves and limited between the LE and TE. Each of them was circumferentially parallel to the camber curve and controlled by 9 nodes, labeled with $\boldsymbol{b}_{l j}(1 \leq l \leq 6$ and $1 \leq j \leq 9)$. Among them, $\boldsymbol{b}_{l l}, \boldsymbol{b}_{l 2}, \boldsymbol{b}_{l 8}$ and $\boldsymbol{b}_{l 9}$ (marked blue in Fig.6a) were fixed to the original hub ( $x=0$ plane) to ensure a smooth transition of the end wall contour, while the rest nodes (marked white) were able to vary freely within $-2.5 \sim+2.5 \%$ span

Figure $6 \mathrm{~b}$ gives the projection of curve and $_{1}$ curve $_{3}$ on the $x-z$ plane as an example. According to the character of Bezier curve, the polyline of $\boldsymbol{b}_{l l} \sim \boldsymbol{b}_{l 9}$ is actually the convex hull of Bezier curve $e_{l}$ A parameter $t$ is introduced, where $t \in[0,1]$. When the node $\boldsymbol{b}_{l j}$ is moved along the $x$ coordinate by $\delta x_{l j}$, the whole curve will also be altered smoothly with its maximum changing at $t=j / n$, by

$$
\left|\delta x\left(\frac{j}{n}\right)\right|=\delta x_{l j} \mathrm{C}_{9}^{j}\left(\frac{j}{n}\right)^{j}\left(1-\frac{j}{t}\right)^{9-j}
$$

Obviously, the variation of $\boldsymbol{b}_{j}$ and $\delta x(j / n)$ have a linear relation.

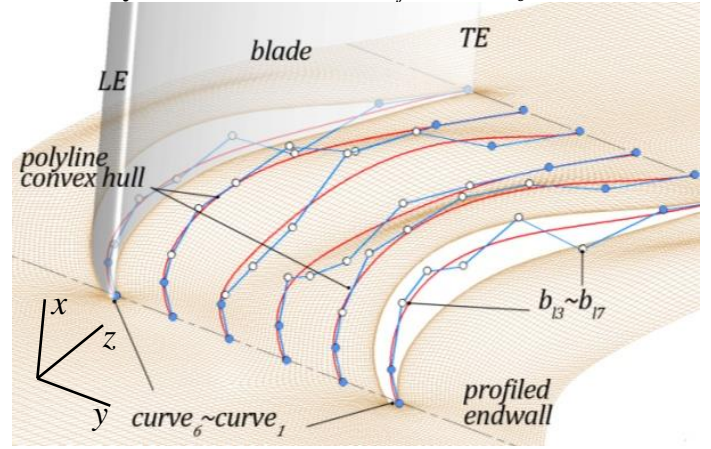

a. end wall surface

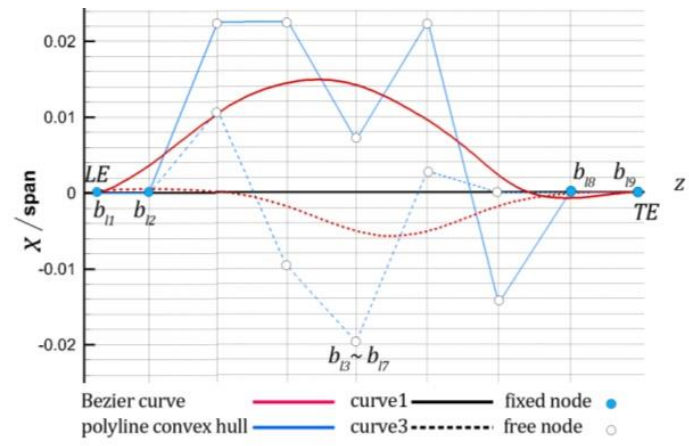

b. projection of curve ${ }_{1}$ on $x-z$ plane

Fig. 6 Contouring method of end wall surface

\subsection{Evaluating the flow field and performance}

The data mining applied in this study employed nine parameters to quantify the secondary flow, blockage and aerodynamic loss of the flow field. The definition of these parameters is as follows.

1) Secondary flow

Two parameters were defined to evaluate the secondary flow in the cascade passage.

$s k e_{T E}$ represents the averaged secondary kinetic energy at the trailing edge $z$-plane. It has been used in Reising's research ${ }^{[9]}$ and defined as

$$
s k e_{T E}=\frac{\iint_{T E} s k e \cdot \rho \boldsymbol{V} d \boldsymbol{A}}{\iint_{T E} \rho \boldsymbol{V} d \boldsymbol{A}}=\frac{\iint_{T E} \frac{1}{2} \rho\left[\boldsymbol{V}-\left(\boldsymbol{V} \cdot \boldsymbol{n}_{\text {prim }}\right) \boldsymbol{n}_{\text {prim }}\right]^{2} \cdot \rho \boldsymbol{V} d \boldsymbol{A}}{\iint_{T E} \rho \boldsymbol{V} d \boldsymbol{A}}
$$

where $\left[\boldsymbol{V}-\left(\boldsymbol{V} \cdot \boldsymbol{n}_{\text {prim }}\right) \boldsymbol{n}_{\text {prim }}\right]$ means the velocity component perpendicular to the direction of primary flow $\left(\boldsymbol{n}_{\text {prim }}\right)$, i.e. secondary velocity. A high value of ske commonly indicates the existence of strong cross and climb secondary flow, or significant flow turning induced by blockage. The mass flow averaged ske at the exit plane thus represents the overall intensity of secondary flow.

Another parameter is the averaged vortex flux at TE:

$$
V o_{T E}=\left|\frac{\iint_{T E} \omega \cdot \operatorname{Ste}\left(-\omega_{z}\right) d \boldsymbol{A}}{\iint_{T E} d A}\right|
$$

in which "Ste $(x)$ " is the unit step function defined as

$$
\operatorname{Ste}(x)=\left\{\begin{array}{l}
1, x>0 \\
0, x \leq 0
\end{array}\right.
$$

According to the definition in equation (3) and the coordinate system illustrated in Fig.1, $V o_{T E}$ only refers to the vortex that has a negative $z$ component, i.e. the same direction as the passage vortex in the TE-plane. Since the passage vortex occupies a dominant role in the vortex system, a high value of $V O_{T E}$ commonly indicates a strong passage vortex developing in the cascade.

2) Blockage 
The evaluation of blockage employs the concept of $A V D R$, i.e. the ratio of pitch averaged TE $V_{z} \rho$ and inlet $V_{z} \rho$ at the same span to evaluate the blockage of the cascade. In the ideal situation, where there is no blockage in the cascade, the meridian streamline will keep parallel to the inlet flow and therefore $A V D R$ equals 1 at full-span range. However, for flow with three-dimensional separation in the suction-hub corner, the local streamline will be commonly blocked and then forced to a different span location. Hence, the out flow will be adjusted to a nonuniform distribution, leading to a lower value of $A V D R$ downstream of the blocked span range. Thus, the blockage level can be evaluated using the normalized deviation of $A V D R$, i.e.

$$
B=\sqrt{\frac{1}{0.5 \text { span }} \int_{0}^{0.5 \text { span }}\left(A V D R(x)-A V D R_{\text {savg }}\right)^{2} d x}
$$

where "savg" represents the span-wise averaged value. A high value of $B$ indicates a significant blockage near the end wall region. In particular, for the stator of a compressor stage, this parameter is likely to be associated with the aerodynamic stability of the upstream rotor.

3) Total pressure loss

A total of six parameters were defined to evaluate the total pressure loss of cascade.

Loss $_{\text {out }}$ reflects the overall loss of the cascade. It is defined as the normalized total pressure difference between the outlet plane and inlet plane, i.e.

$$
\operatorname{Loss}_{\text {out }}=\frac{\iint_{\text {out }} \frac{P t_{\text {in,mavg }}-P t(x, y)}{(P t-P)_{\text {in,mavg }}} \rho \boldsymbol{V d A}}{\iint_{\text {out }} \rho \boldsymbol{V} d \boldsymbol{A}}
$$

in which the subscript 'in', 'out' and 'mavg' respectively represents inlet plane, outlet plane and the mass flow averaged value.

Evaluation for loss sources is by a quantitative analysis model derived in our previous work ${ }^{[13]}$. According to the model, the blade passage can be divided into four sub-regions upon the mechanism of loss generation, as shown in Fig.7. Each of them corresponds to a different loss source and is quantified by a normalized volume integral of the dissipation function, i.e.

$$
C p_{\text {source }}=\frac{-\Delta P t_{\text {source }}}{(P t-P)_{\text {in,mavg }}}=\frac{\iiint_{\text {region }} \Phi d x d y d z}{(P t-P)_{\text {in,mavg }} \iint_{\text {in }} \boldsymbol{V} d \boldsymbol{A}}
$$

Four sources of loss were considered: $C p_{U}, C p_{P}, C p_{F R I}$ and $C p_{D}$.

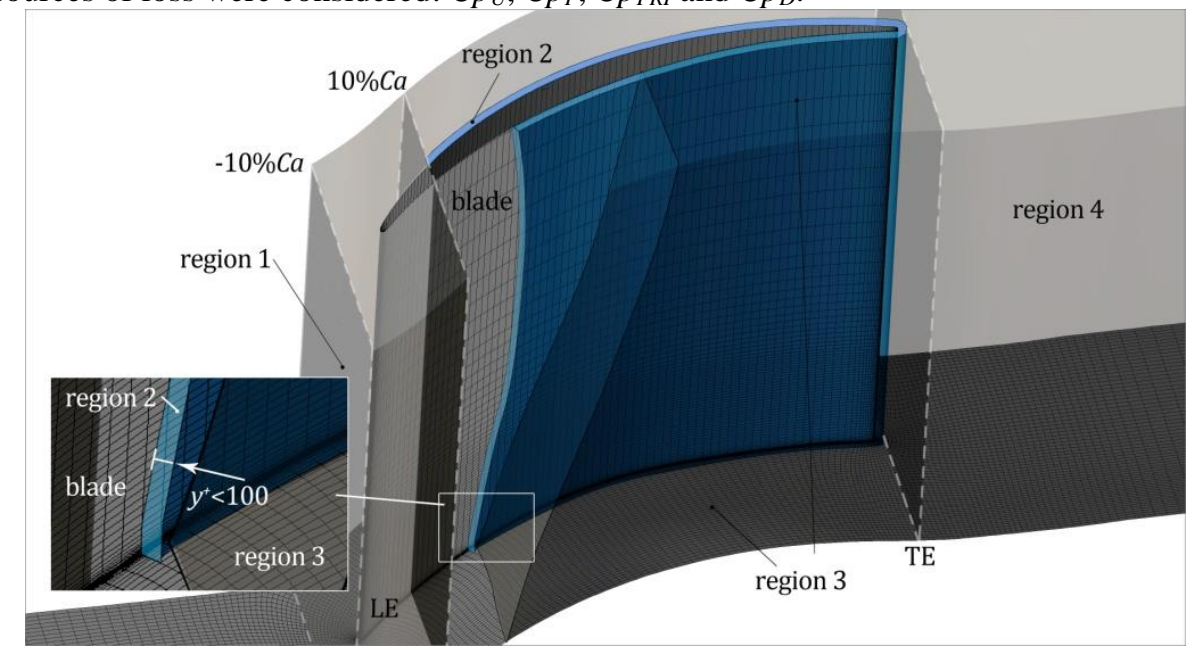

Fig.7 division of passage for loss sources evaluation

$C p_{U}$ corresponds to region 1 . The loss in total pressure comes mainly from the shear effect induced by the LE turning of the flow.

$C p_{F R I}$ is calculated in region 2 , which is a skin-like layer over the blade surface within $y^{+}=100$. The total pressure loss comes mainly from the surface friction.

$C p_{P}$ is calculated in region 3, which occupies the rest of the space from 10\% 100\% Ca apart from region 2 . In this region, $P t$ loss mainly comes from the shear effect induced by corner separation.

$C p_{D}$ is the loss coefficient in region 4 , which occupies the space downstream of the TE. The loss source in this region mainly corresponds to the dissipation of low energy fluid (induced by the upstream separation) coupled with the dissipation of secondary flow coming out of the passage.

In view of the mechanism of the above four sources, the loss purely associated with corner 
separation can be approximately evaluated by removing the dissipation of secondary flow from the sum of $C p_{D}$ and $C p_{P}$. This produces another loss parameter

$$
C p_{S E P}=C p_{D}+C p_{P}-\frac{\Delta s k e_{\text {mavg }}}{(P t-P)_{\text {in,mavg }}}
$$

where $\Delta s k e_{\text {mavg }}$ represents the reduction of mass flow averaged secondary kinetic energy from the TE to outlet of the computational domain, i.e. the major part of the dissipation of secondary flow.

So far, all the parameters for the quantitative analysis, i.e., ske $e_{T E}, V_{T E}, B, \operatorname{Loss}_{\text {out }}, C p_{U}, C p_{P}, C p_{F R I}$, $C p_{D}$ and $C p_{S E P}$, have been defined. A grid dependence study for the parameters was also applied for the flat end wall cascade to make sure the CFD method have sufficient accuracy even for the detailed flow field analysis, as shown by table 2. G2 are the grids employed in this study. Compare with G2, G1 grids do not refine the nodes near end wall region and the grids size totals up to 1.2 million; G3 grids furthermore refine the nodes in the region of higher span and the grids size is about 2.3 million. Table 2 show the relative change of four types of parameters for G1, G2 and G3. It is seen that the relative change of all four types of parameters are below $0.55 \%$ when $\mathrm{G} 2$ is further refined. The grids employed in this study thus have sufficient accuracy for the detailed flow field analysis.

Tab.2 Grid dependence study for flow field parameters

\begin{tabular}{|c|c|c|c|c|c|c|}
\hline $\begin{array}{l}\text { work } \\
\text { point }\end{array}$ & $\begin{array}{c}\text { performance } \\
\text { parameters }\end{array}$ & G1 grids & $\mathrm{G} 2$ grids & $\begin{array}{l}\text { relative change } \\
\text { from } \mathrm{G} 1 \text { to } \mathrm{G} 2\end{array}$ & G3 grids & $\begin{array}{l}\text { relative change } \\
\text { from } \mathrm{G} 1 \text { to } \mathrm{G} 2\end{array}$ \\
\hline \multirow{4}{*}{$\begin{array}{l}\stackrel{0}{1} \\
\text { II } \\
\therefore \sim\end{array}$} & Loss $_{\text {out }}$ & 0.09643 & 0.09151 & $-5.10 \%$ & 0.09151 & $0.00 \%$ \\
\hline & $s k e_{T E}$ & 1.762 & 1.823 & $3.46 \%$ & 1.817 & $-0.33 \%$ \\
\hline & $B$ & 0.1300 & 0.1381 & $6.23 \%$ & 0.1387 & $0.43 \%$ \\
\hline & $V o_{T E}$ & 125.1 & 131.2 & $4.88 \%$ & 130.8 & $-0.31 \%$ \\
\hline \multirow{4}{*}{$\stackrel{\circ}{\stackrel{\circ}{\text { II }}}$} & $\operatorname{Loss}_{\text {out }}$ & 0.1699 & 0.1624 & $-4.41 \%$ & 0.1615 & $-0.55 \%$ \\
\hline & $s k e_{T E}$ & 1.791 & 1.592 & $-11.11 \%$ & 1.589 & $-0.02 \%$ \\
\hline & $B$ & 0.2532 & 0.2458 & $-2.92 \%$ & 0.2458 & $0.00 \%$ \\
\hline & $V o_{T E}$ & 115.3 & 113.4 & $-1.65 \%$ & 113.8 & $0.35 \%$ \\
\hline
\end{tabular}

\subsection{Results and discussion}

This study employed a normalized correlation factor to quantify the correlation between parameters. It was known that the cascade performance is actually an abstract function of profiling parameters, i.e.

$$
\left(s k e_{T E}, V o_{T E}, B, \text { Loss }_{\text {out }}, C p_{U}, C p_{P}, C p_{F R I}, C p_{D}, C p_{S E P}\right)^{T}=\boldsymbol{F}\left(\begin{array}{cccc}
x_{13} & x_{14} & \cdots & x_{17} \\
x_{23} & x_{24} & \cdots & x_{27} \\
\vdots & \vdots & & \\
x_{63} & x_{64} & & x_{67}
\end{array}\right)
$$

where $x_{i j}$ is the $x$ coordinate of $\boldsymbol{b}_{i j} . p_{1}$ and $p_{2}$ were used to represent a pair of the above parameters, either two performance parameters or a performance parameter coupled with a profiling parameter. The normalized correlation factors between $p_{1}$ and $p_{2}$ could then be calculated by

$$
\gamma\left(p_{1}, p_{2}\right)=\frac{\iint\left(p_{1}-\overline{p_{1}}\right)\left(p_{2}-\overline{p_{2}}\right) d p_{1} d p_{2}}{\sqrt{\int\left(p_{1}-\overline{p_{1}}\right)^{2} d p_{1}} \sqrt{\int\left(p_{2}-\overline{p_{2}}\right)^{2} d p_{2}}}
$$

Using the Monte Carlo estimate, it became

$$
\gamma\left(p_{1}, p_{2}\right)=\frac{\sum_{k=1}^{n}\left(p_{1}{ }^{k}-\overline{p_{1}}\right)\left(p_{2}{ }^{k}-\overline{p_{2}}\right)}{\sqrt{\sum_{k=1}^{n}\left(p_{1}{ }^{k}-\overline{p_{1}}\right)^{2}} \sqrt{\sum_{k=1}^{n}\left(p_{2}{ }^{k}-\overline{p_{2}}\right)^{2}}}
$$

Thus, a sampling in the design space was required to perform the correlation analysis.

The variable interval of $x_{l j}$ was divided to 10 discrete levels. A random combination was then applied for the profiling parameters and generated a sampling set of over 600 different non-axisymmetric end wall cases. Their performance parameters were then obtained by applying simulation. Their correlation factors were calculated through equation (11) and finally listed in Table 3 . The top right part gives the result at the design point while the bottom left part gives the result of the near stall point. Note that the correlation factor $\gamma\left(p_{1}, p_{2}\right)$ reveals how the first parameter $p_{1}$ is relevant to second one $p_{2}$ during profiling. For random variables, it is always the case that $|\gamma| \leq 1$. The more $|\gamma|$ approaches 1 , the closer $p_{1}$ and $p_{2}$ are relevant with each other. The positive/negative sign of $\gamma$ generally indicates a synchronous/opposite variation of $p_{1}$ and $p_{2}$. In particular, $\gamma=0$ means that $p_{1}$ and $p_{2}$ are independent with each other, while $\gamma= \pm 1$ means $p_{1}$ has a positive/negative linear relation with $p_{2}$. Thus, the influence of end wall contouring on the variation of loss, blockage, secondary flow and the passage 
vortex can be revealed.

Tab.3 Correlation factors of flow parameters

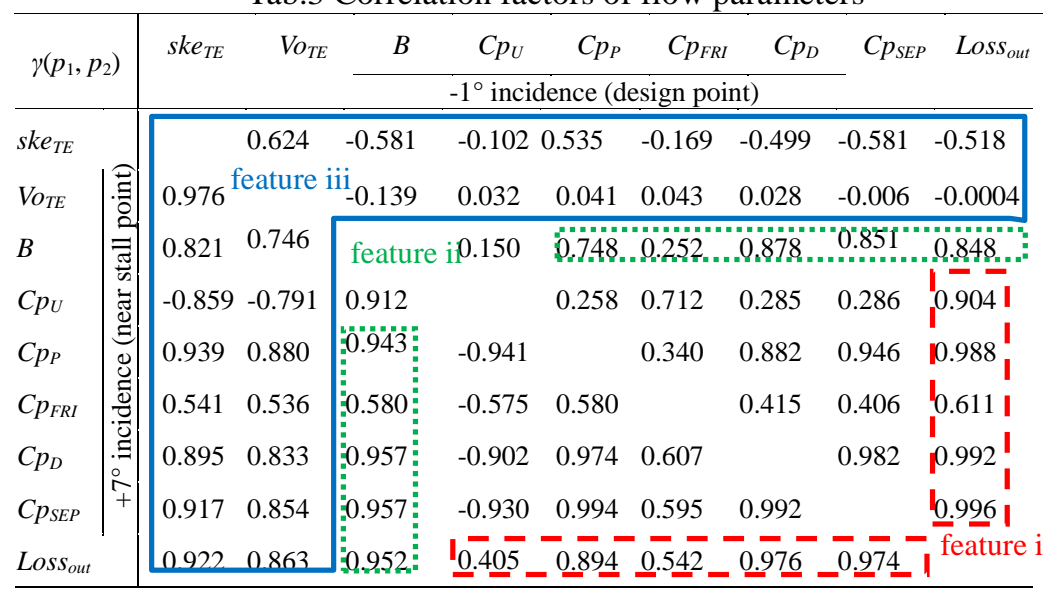

The following features, labeled with different frames in Table 3, should be noted:

i. The influence of the PEW on the corner separation plays a major role in varying the overall loss over the full operating range. The variation of the loss sources downstream of the TE has a larger effect than that within the passage at the design point but a roughly equal effect at the near stall point;

ii. the variation of blockage has an almost linear relation with the corner separation loss in the full operating range;

iii. at the design point, the total pressure loss varies approximately inversely with the overall secondary flow intensity, and is almost independent from that of the passage vortex. Near stall point, however, the variation of total pressure loss and secondary flow intensity show a synchronous variation. Both of them are more closely related to the variation of passage vortex.

The first two features coincide with the literature study. The former additionally points out the different influences of PEW on the upstream and downstream loss sources at different throttling. This would indicate that the development of PEW should focus more on reducing the loss sources downstream of passage at design point, and take in to account the in-passage loss sources as well near stall point.

The third feature, however, provides a complex relationship between the variation of loss and secondary flow which seldom been mentioned by the previous studies. Since it is a consideration of this paper, detailed discussion now follows to clarify its mechanism.

A general illustration for cascade secondary flows is given according to their relation with loss, as sketched in Fig. 8 for a better understanding. The blade passage can be generally divided into three sub-regions, labeled region 1,2 and 3. The end wall cross-passage flow in region 1 is known as the basic cause for corner separation ${ }^{[1]}$. Stronger secondary flow in this region leads to higher total pressure loss. In region 2, the climb flow along the SS is found to give a pre-mixture for the low energy fluid before it meets the primary flow ${ }^{[13]}$, and thus helps reduce loss and relieve corner separation. Secondary flow in region 3 represents the derivation of primary flow and is passively produced because of the blockage of the corner separation. Its intensity can be influenced by any factor associated with corner separation, including the previously mentioned cross and climb flows.

The reason why overall secondary flow shows different correlation with overall loss at different operating points would likely come from the variation of secondary flow in the above three sub-regions. Some typical cases are chosen from the sampling set for a more detailed explanation.

Figure 9 provides the Loss $_{\text {out }}-s k e_{\mathrm{TE}}$ map for all samples in the database. Six typical cases are chosen to cover the variation of loss and secondary flow intensity - four of them for the design point, label a-d in Fig.9a, and the other two for near stall point, labelled e and f in Fig.9b.

At the design point, cases $\mathrm{b}$ and $\mathrm{d}$ show different $s k e_{\mathrm{TE}}$ but both have significant lower total pressure loss than cases a and c. According to the through-flow loss and secondary flow, which are evaluated using $\Phi_{\text {avg }}$ and $s k e_{\text {mavg }}$ in Fig. 10, the lower loss is associated with their lower $\Phi_{\text {avg }}$ between $0.5 C a$ and the outlet. Their $s k e_{\text {mavg }}$ curves present higher or lower absolute values, however, both include a much slower "rise and drop" than cases a and c. 


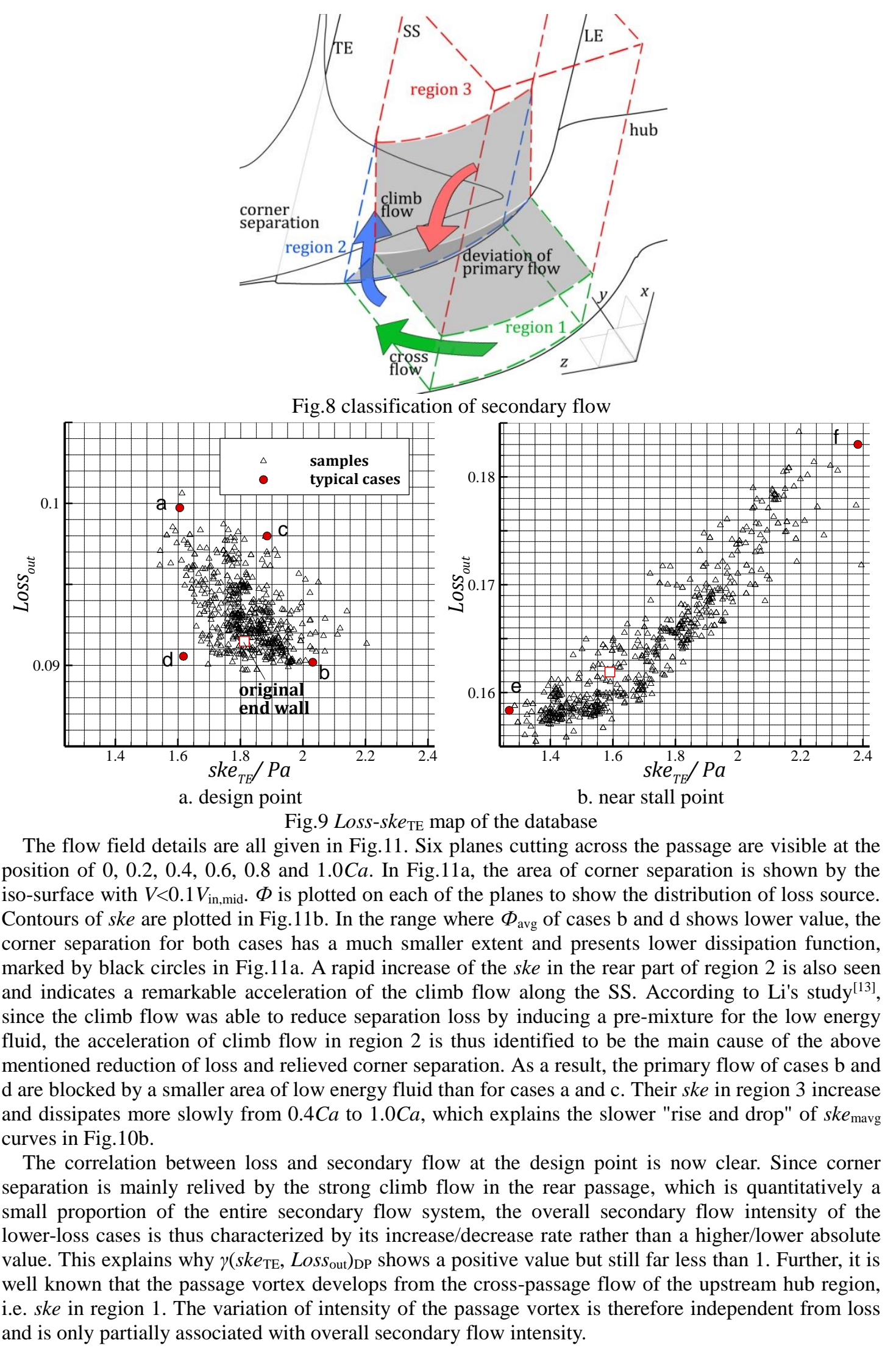



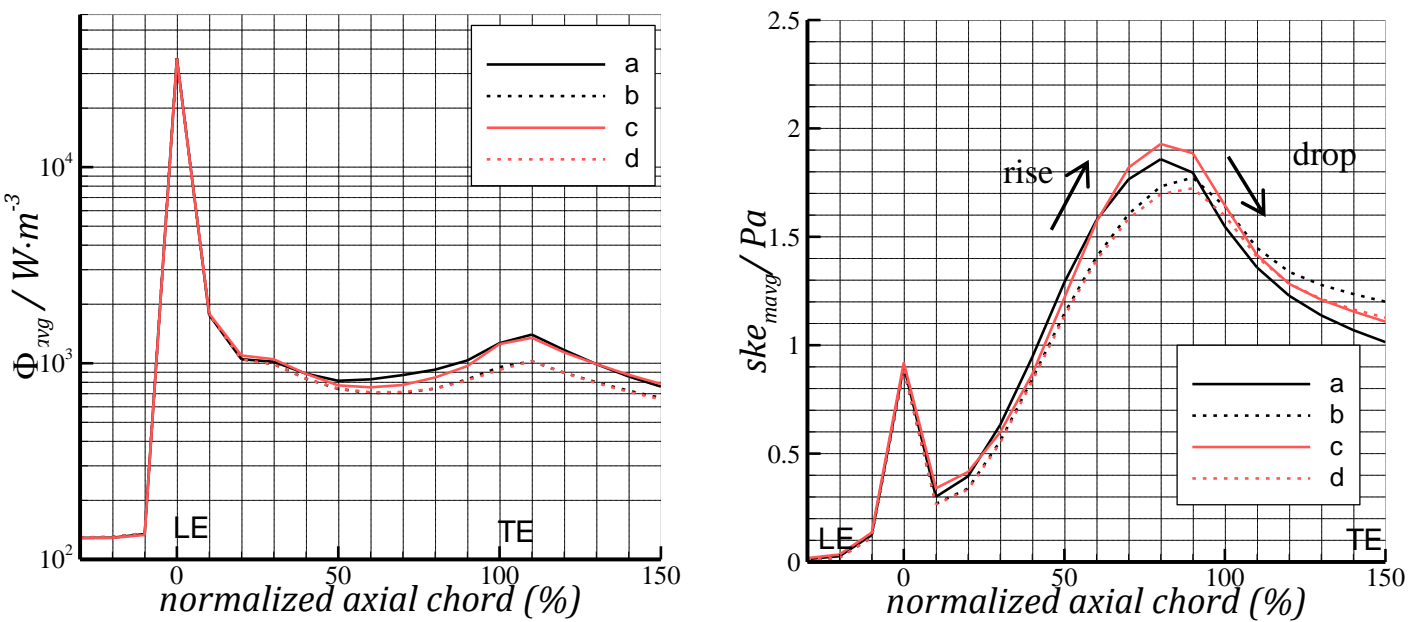

a. area-averaged dissipation function

b. massflow-averaged ske

Fig.10 streamwise development of loss and secondary flow at design point

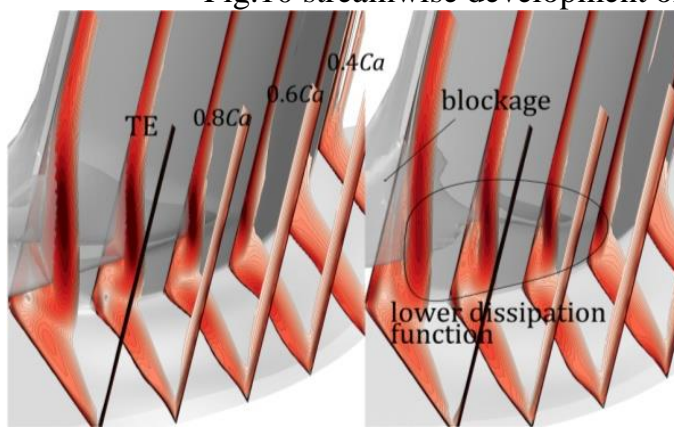

case a

case b

case $\mathrm{c}$

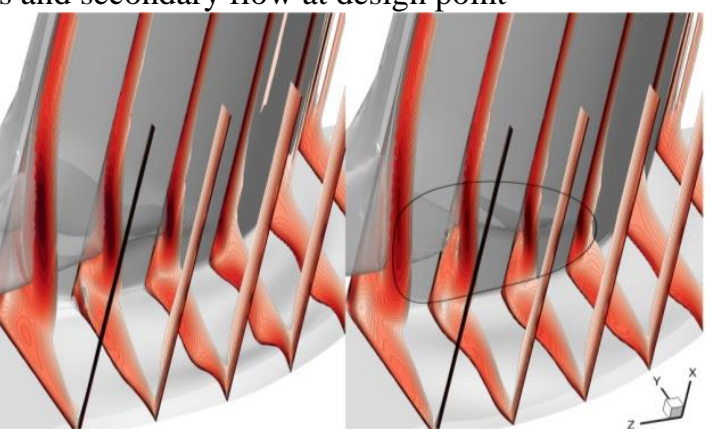

case d

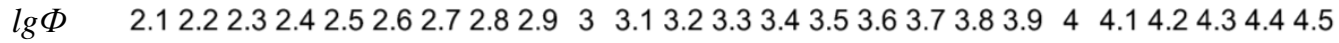

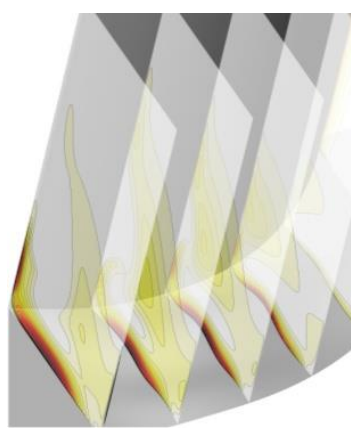

case a

a. corner separation and dissipation function

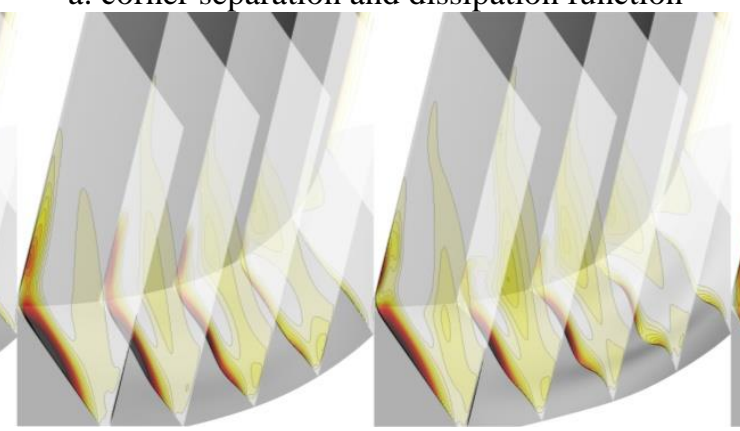

case $b$

case c

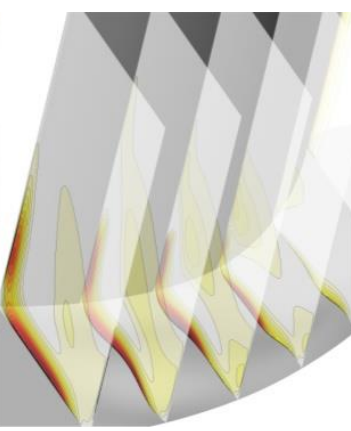

case $\mathrm{d}$

ske $(\mathrm{Pa})$

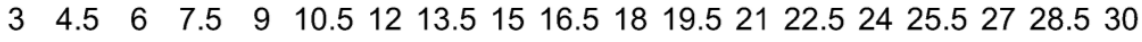

b. secondary flow

Fig.11 three-dimensional flow field at design point

Compared with the design point, the PEW shows quite another story when it operates near the stall point, as shown by the typical cases e and $\mathrm{f}$ in Fig.9b, Fig.12 and Fig.13. Both of $s k e_{\text {mavg }}$ and $\Phi_{\text {avg }}$ in case $\mathrm{f}$ show remarkably higher values than for cases e from a much more upstream location around $0.1 \mathrm{Ca}$. Correspondingly, the ske contour of case f sees higher distribution of ske than case e in all three regions throughout the passage. Since $\Phi$ contours of case f show much more serious corner separation and higher dissipation function upstream of $0.6 \mathrm{Ca}$ than case e, the hub cross flow in the front region 1 is inferred to act as the dominant contributor. The correlation between loss and cross-passage flow should be just as those concluded in the previous studies ${ }^{[1,4-7]}$, that stronger cross flow in the front end wall intensifies the accumulation of low energy fluid and the corner separation, thus leading to subsequent higher dissipation later in the passage. As a result, the primary flow incurs greater deviation. Higher ske also appears in region 3 in the front part of passage.

The main reason for the changes between the design point and near stall point is mainly associated 
with the development of the corner separation. Under a higher incidence, corner separation initiates in a more upstream region and occupies a larger area than at design point, as shown in Fig.13. The significant development of the corner separation makes the deviation secondary flow take a higher proportion in all three high ske regions. Since it is induced by the hub cross flow, the significant corner separation actually "amplifies" the proportion of hub cross flow in the whole secondary flow system. As a result, the two components of secondary flow, i.e. cross flow and deviation flow become more closely related with each other, which also increase their correlation with corner separation. The development of the passage vortex is also more closely related to the loss and secondary flow via the dominant cross-passage flow in the overall secondary flow system.

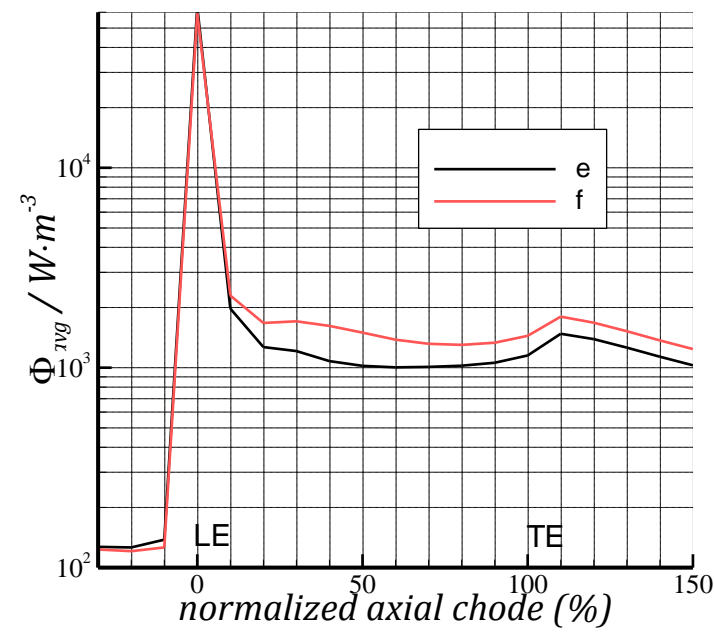

a. area-averaged dissipation function

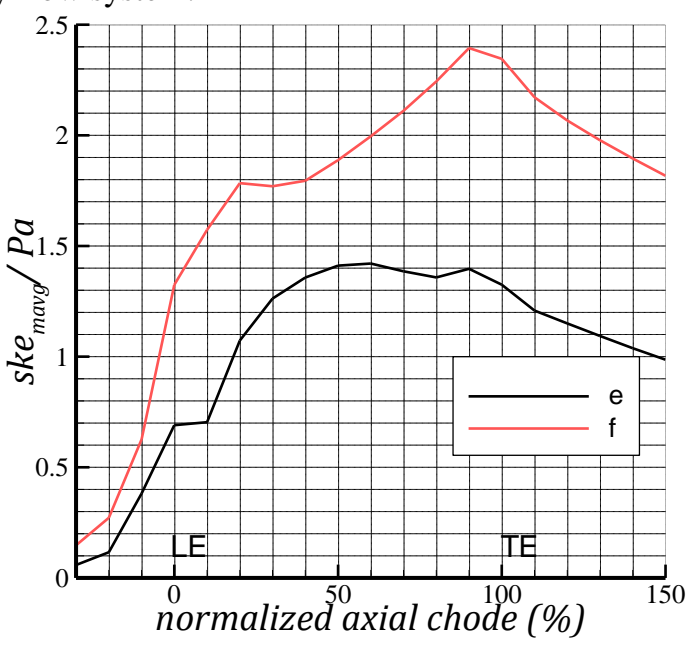

b. massflow-averaged ske

Fig.12 streamwise development of loss an secondary flow near stall point

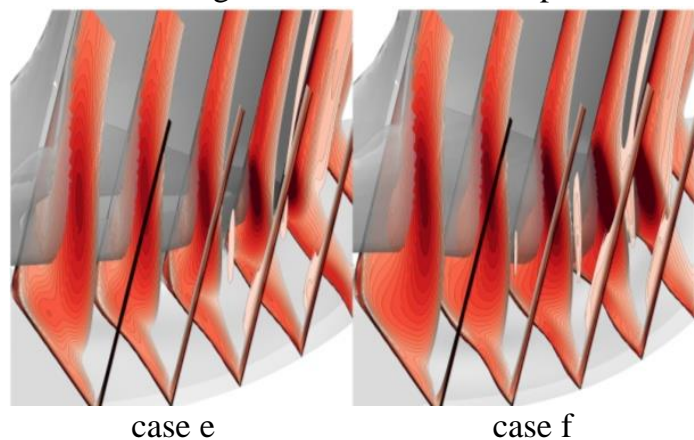

case e case $\mathrm{f}$

ion function

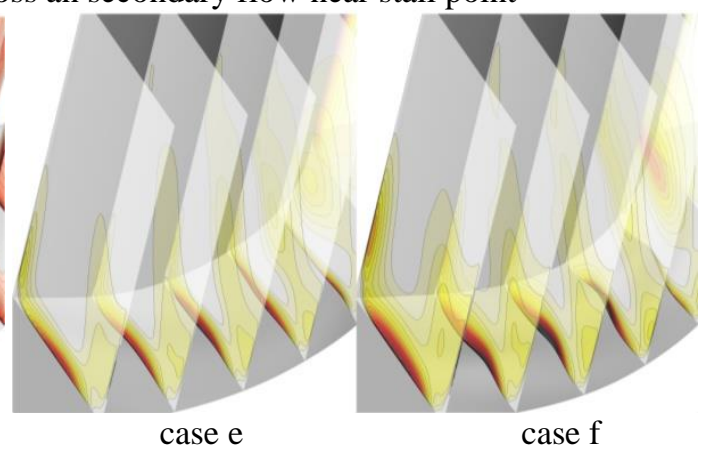

b. secondary flow

Fig.13 three-dimensional flow field near stall point

\subsection{Profiling principles}

So far, the impact of the PEW on the interaction between loss, secondary flow and passage vortex has been presented. The following points can be concluded as the general guidance.

Using PEW, the control of corner separation is anagolous with that of span-wise blockage, and is of most importance for reducing total pressure loss over the full operating range.

At the design point, the impact of PEW is most apparent in the rear part of the passage. The influence of PEW on overall loss shows an approximately inverse relation with its influence on the intensity of the secondary flow. It is almost independent of the passage vortex, because corner separation and the passage vortex are respectively associated with the cross-passage secondary flow and SS climb flow, neither of which contributes a major part of the absolute value of secondary kinetic energy at the TE-plane. It is therefore undesirable to reduce loss at the design point by limiting the development of the passage vortex or by globally reducing the ske.

Near the stall point, the influence of PEW demonstrates a similar level of influence on the front and rear parts of passage. Because of the developing extent of the corner separation for the flow field, which on the one hand increases the influence of the hub cross flow among all three secondary flow 
components, on the other hand closely associates the overall secondary flow with corner separation. The overall loss, the intensity of the overall secondary flow and the passage vortex almost change in the same direction Therefore, near the stall point or the point at which significant corner separation exists in the passage, reduction of loss can be accomplished by limiting the development of the passage vortex or reducing the overall secondary flow.

The following passage will focus on the second aspect identified in section 1, i.e. the effective methods by which end wall shaping controls the cascade performance and flow field.

\section{Effective profiling rules using non-axisymmetric end wall}

A multi-objective optimization was applied to establish the most effective profiling rules for compressors. However, the traditional analysis of rising and depression of end wall surface is not used here because, as pointed out in the Introduction section, the modification of the pressure distribution produced by PEW vary substantially with a change of inflow condition, thus making the profiling rules transferrable between different operation points and not applicable among different compressors (cascades). To solve this problem, a quantitative analyzing tool was employed from previous work by the author ${ }^{[13]}$, in which the geometry feature is not the main focus, instead the influence of profiling is treated as a "correctional force" that changes the original flow field to a better PEW flow field. The flow near the hub end wall is governed by

$$
-\frac{1}{\rho} \nabla P_{c o r r} \approx \boldsymbol{V}_{\text {ori }} \cdot \boldsymbol{\nabla} \boldsymbol{V}_{\text {corr }}+\boldsymbol{V}_{\text {corr }} \cdot \nabla \boldsymbol{V}_{\text {pew }}=\left\{\begin{array}{l}
\boldsymbol{V}_{\text {ori }} \cdot \nabla \boldsymbol{V}_{\text {corr }}, V_{\text {ori }}>>V_{c o r r} \\
\boldsymbol{V}_{\text {corr }} \cdot \nabla \boldsymbol{V}_{\text {corr }}, V_{\text {ori }}<<V_{\text {corr }}
\end{array}\right.
$$

where quantities with the subscripts "ori", "pew" and "corr" respectively represent the values at a flat end wall, profiled end wall and its variation induced by PEW, i.e. $Q_{c o r r}=Q_{p e w^{-}} Q_{o r i}$. Note that equation (14) is a $y-z$ vector equation. The left side parameter, i.e. the negative produc of $y$ - $z$ correctional pressure gradient and density shares the same dimension with acceleration and approximately acts as the driving force for the value of convective acceleration of $V_{\text {corr }}$. Thus, $-\frac{1}{\rho} \nabla P_{c o r r}$ is used as a tool for analyzing the correctional force induced by PEW and is termed the "driving force" in the following passages for convenience.

\subsection{Optimization of PEW}

To demonstrate better performance, a compressor stage is expected to have higher efficiency at design point and higher $\pi_{\mathrm{NS}} / m_{\mathrm{NS}}$ near stall point. Therefore, the stator row should have lower loss at both operating points to reach a higher total pressure ratio and reduced tip blockage near stall to reduce the risk of an earlier rotating stall of the rotor. Note that the data mining has confirmed that Loss out $_{\text {t }}$ and $B$ changes in the same direction near stall point during profiling. Thus, there is no need to set $B_{\mathrm{NS}}$ as an objective function. A multi-objective optimization aimed at minimizing Loss $_{\text {out }}$ at the design point and near the stall point will be thorough enough to achieve an effective profiling for both efficiency and stability of the compressor.

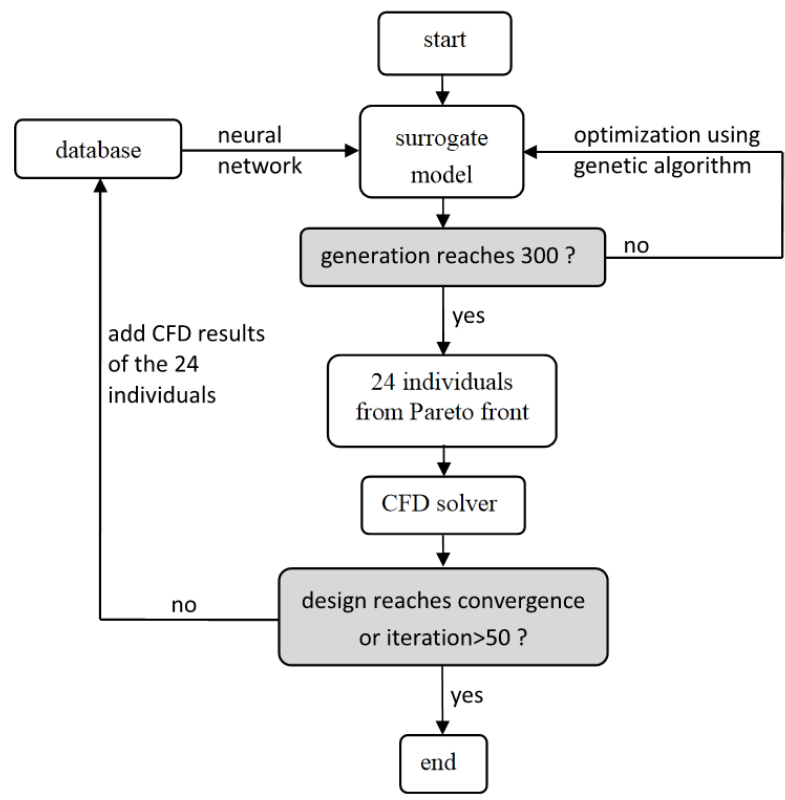

Fig.14 Framework of the optimization

Figure 14 gives the framework of the optimization process used for the PEW. It comprised two layers of iteration. The inner one was the optimization iteration, which was formed by a radial basis 
function neural network as the surrogate CFD solver and a genetic algorithm as the optimizer. The neural network was initially trained by the samples previously used for data mining, establishing the projection from the 30 profiling parameters to $\left(\text { Loss }_{\text {out }, \mathrm{NS}}, \text { Loss }_{\text {out } \mathrm{DP}}\right)^{\mathrm{T}}$. Then a multi-objective genetic algorithm was applied with the NSGA-II searching strategy and progressed through a 300-generation evolution. 24 individuals were finally selected at uniform spacing along the Pareto front as the optimum results. Thereafter, CFD simulation was applied for the 24 individuals to predict their results. The 24 PEWs were added to the sampling database to refine the neural network, and then a new outer iteration was started.

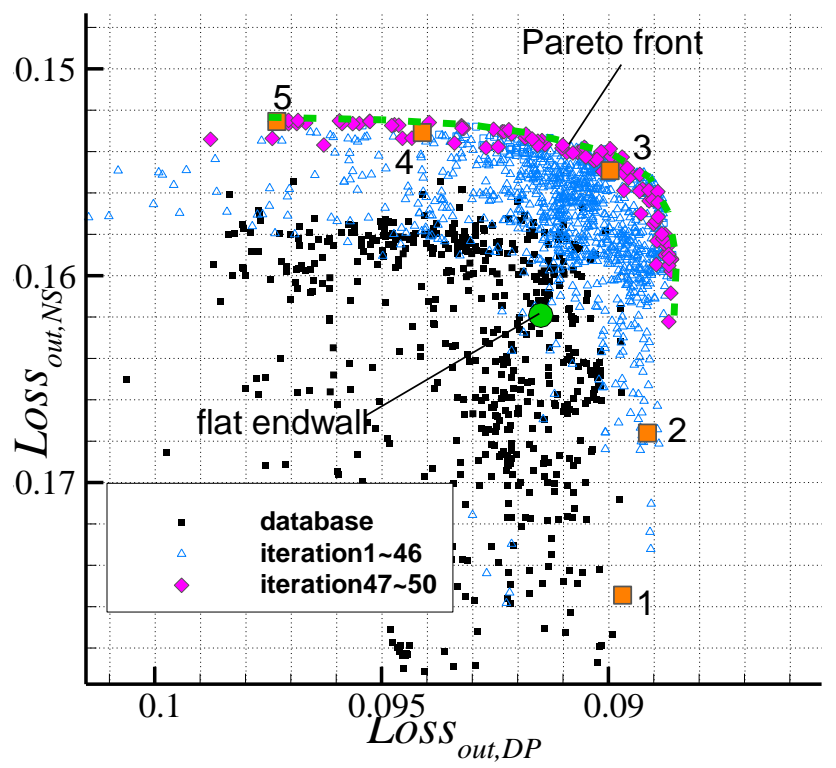

Fig.15 Loss $_{\text {out }, \mathrm{NS}}-$ Loss $_{\text {out }, \mathrm{DP}}$ projection during optimization

Figure 15 gives the Loss $_{\text {out }, \mathrm{DP}}-$ Loss $_{\text {out }, \mathrm{NS}}$ projection from the optimization process. The case with a flat end wall is marked by a green solid circle in the map. Other cases belong to the original database (black solid squares) or the optimization iterations (blue triangles and pink diamonds). After proceeding for 46 steps, the optimization profiling reached convergence and finally distributed around the top-right corner of the map, with the Pareto front approaching the shape of a right angle. This indicates that there is not much conflict between minimizing Loss $_{\text {out }, \mathrm{DP}}$ and Loss $_{\text {out }, \mathrm{NS}}$. Therefore, the optimum control of end wall flow is generally independent for design and near stall points.

Five individuals were typically selected from Fig. 15 to conclude the optimum profiling principle, marked by orange squares and labeled 1-5. Among them, cases 1 and 2 emphasize the performance at the design point. Cases 3-5 are chosen near the Pareto front, with case 4 and 5 representing the advantageous cases for NS and case 3representing a balanced optimization of both operating points.

\subsection{Data mining for profiling parameters}

Since the perturbation of the non-axisymmetric end wall was limited by a prescribed amplitude $( \pm 2.5 \%$ span $)$ and range (from LE to TE), the result of optimization was only a compromised result and not every detail of the optimum end wall was associated with a reduction of loss. To identify the related area of the end wall surface where PEW exerts its influence, a correlation analysis for performance parameters and profiling parameters was applied in the first instance with the same theory and samples as those used in section 3 .

Figure16 gives the maps of $\gamma\left(\operatorname{Loss}_{\text {out }}, x_{l j}\right)$ for both the design and near stall points. Although the end wall surface was generated with Bezier curves, whose change is strongly influenced by the combined action of all the control points, the local deflection of each curve was still linearly related with the variation of each control point, as shown in equation (1). Thus, a higher value of $\mid \gamma\left(\right.$ flow parameter, $\left.x_{l j}\right) \mid$ indicates a more significant influence of the region on the flow field. Additionally, the profiling parameters are $x$-coordinates of control points $\boldsymbol{b}_{l j}(1 \leq l \leq 6,3 \leq j \leq 7)$ rather than an arbitrary point on the profiling surface. Therefore, the iso-lines are drawn here only for the convenience of comparison, and it is the value of $\gamma$ at a certain control point that is meaningful. 


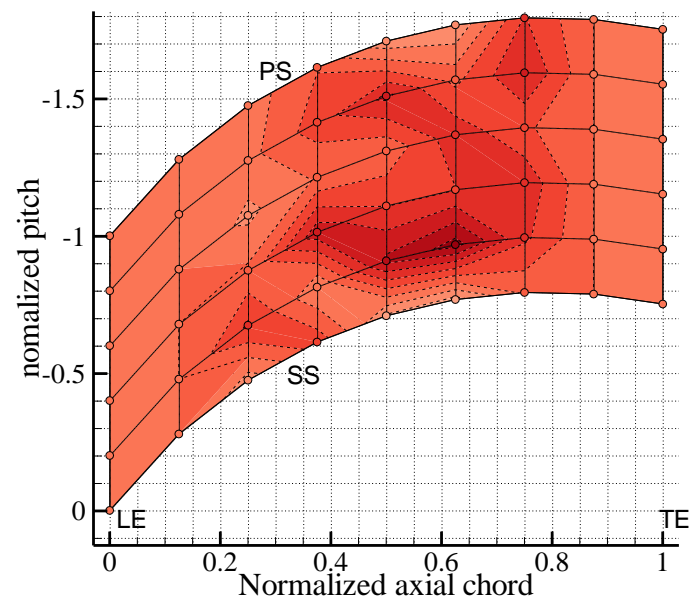

a. correlation map at design point

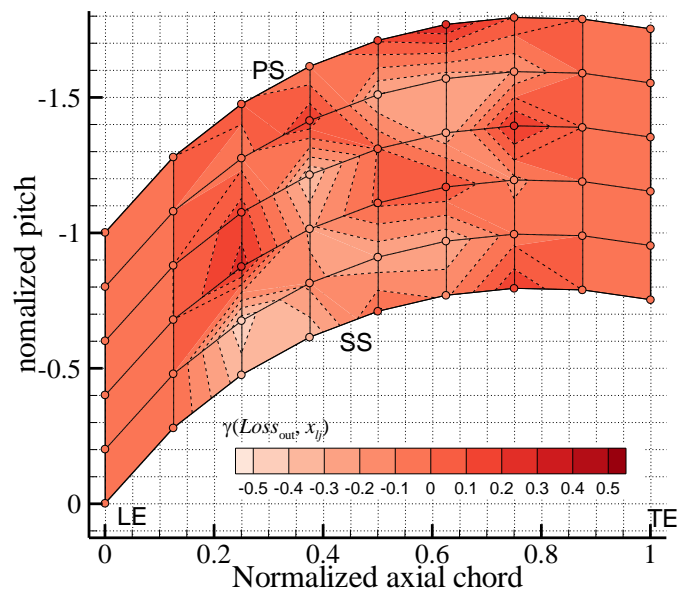

b. correlation map near stall point

Fig.16 Correlation factors between control nodes and flow parameters

At the design point, there are two related regions for loss. One is locates in the SS-half of the passage from $0.1 C a$ to $0.9 C a$, of which the region around $\boldsymbol{b}_{25}$ and $\boldsymbol{b}_{26}$ is inferred to be the most significant part according to its value. The other spreads in the rear region of full pitchwise range. Near the stall point, three related regions are found. The most significant one is located along the front SS corner around $\boldsymbol{b}_{12}$, $\boldsymbol{b}_{13}$ and $\boldsymbol{b}_{14}$. The other two occupy the center and rear parts of passage, roughly separated by $\boldsymbol{b}_{54}-\boldsymbol{b}_{45}-\boldsymbol{b}_{36}$. 4.3 Effective profiling rules

Figure 17 gives the distribution of $-\frac{1}{\rho} \nabla P_{\text {corr }}$ for the optimum cases, i.e. case $1-5$, of the multi-objective optimization. Arrows illustrate the direction and contours provide the magnitude. A high value of driving force indicates a strong correctional force exerted on the end wall velocity. The analysis will focus on the relevant regions that have been identified, marked by dash lines and labeled using Roman numerals. Additionally, since previous analysis clearly confirmed that the reduction of loss mainly results from the relieved corner separation, priority will be given to this mechanism during the discussion.

1) Design point

At the design point, the driving force in Region I shows remarkable variation between the five cases. The correlation analysis also shows it to be the most important region for the control of corner separation.

For the two low-loss cases, i.e. case 2 and 3, Region I can be divided into three sub-regions from outside to inside, marked 1 3, where the direction of the driving force goes against, towards and then against the cross-passage flow. The reverse force in I-1 extends to the upstream region near the SS corner and weakens the cross flow from the front SS corner to the rear mid-pitch, thus slowing down the accumulation of low energy fluid from PS. Then the cross flow is locally accelerated towards the SS corner and forced to climb span-wise by the driving force in I-2 and I-3. Since the generation of climb flow in the rear SS corner has been shown to be a positive factor in the correlation analysis, which gives a pre-mixture of low energy fluid in the separation region and reduces loss, the combination of driving force in the rear SS corner, i.e. the towards-against model, seems critical for loss reduction.

Compared with cases 2 and 3, cases 4 and 5 show two distinct differences. One is the front part in I-1. Both of case 4 and 5 have a strong force towards the SS, which could promote the secondary flow in the front region and make the corner separation start early. This is obviously detrimental for cascade flow and is inferred to be the main reason why PEW incurs higher total pressure loss in cases 4 and 5 . Another difference is the combination of the driving forces in I-2 and I-3. Although, cases 4 and 5 show a higher force in region I-2, it is located at a farther distance from SS and segregated by a much wider stripe of complex driving force in I-3. In view of the mechanism of the climb flow, this distribution is is inferred to be not as efficient as in case 2 and 3 to increase the climb velocity of secondary flow at the rear SS corner. Both of the above aspects lead to more serious corner separation and thus higher loss. As for case 1 , it has a similar spatial distribution of driving force in I-3 as cases 4 and 5 , but with a much more extensive area and a higher value of driving force in I-1 and I-2. The combined effect of all three sub regions means that case 1 has a lower loss than cases 3-5, but it is still higher than for case 2 .

According to the above analysis, it can be seen that an effective flow control method at the design point should broadly weaken the development of cross flow from front SS corner to the rear mid-pitch while locally strengthening the climb flow around the rear SS corner. This can be accomplished by a 
combination of "against-towards-against" driving forces along the direction of the hub cross flow in the SS-rear region of the passage.
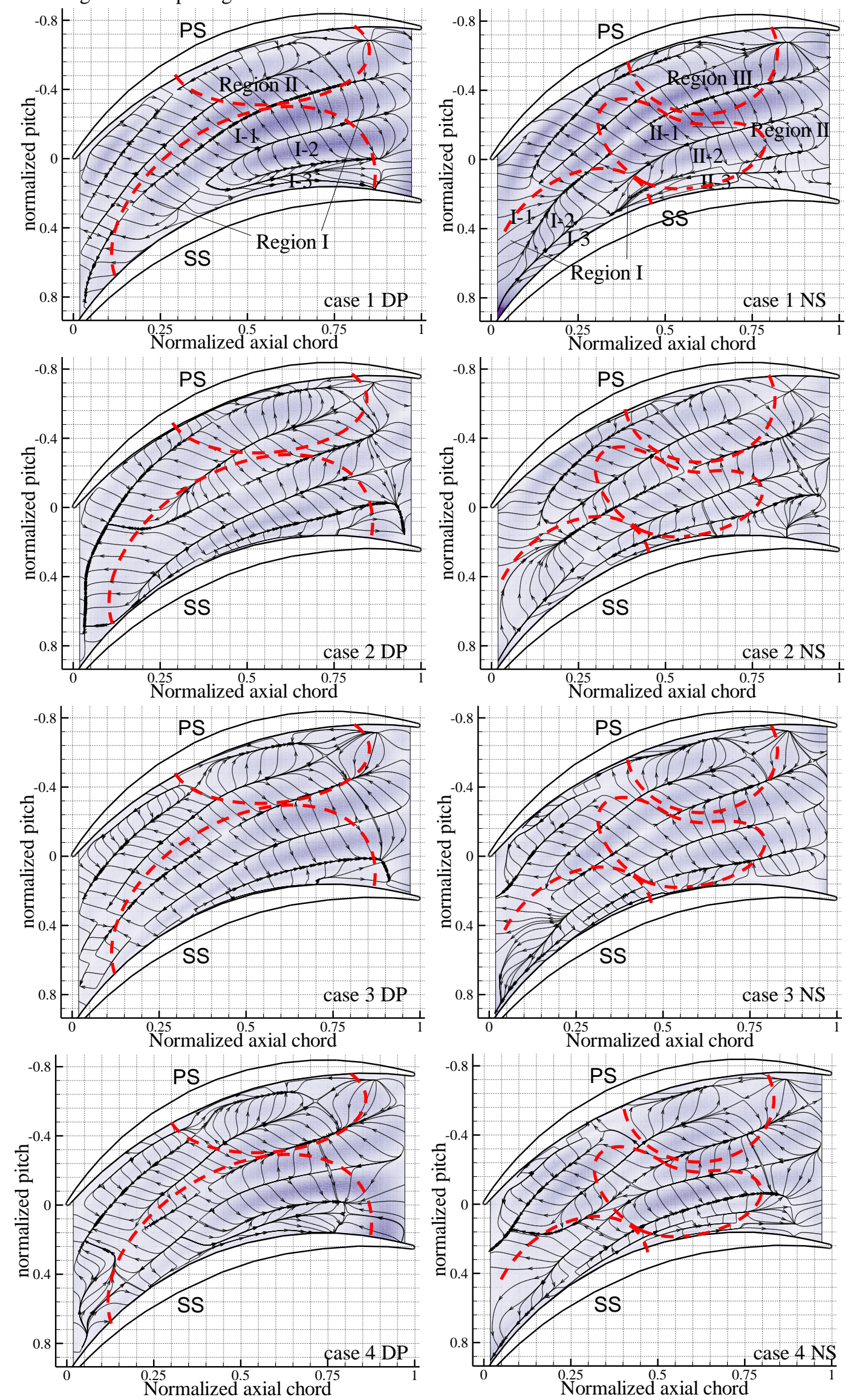

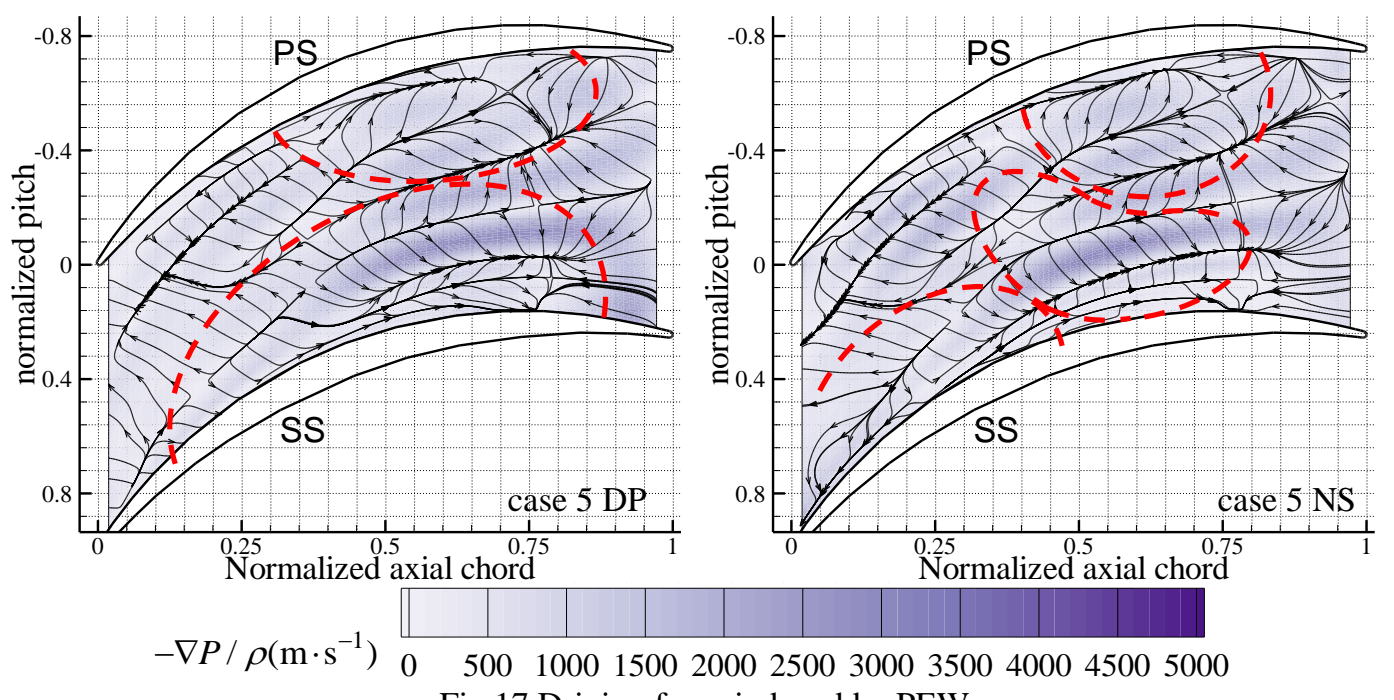

Fig.17 Driving force induced by PEW

2) Near stall point

Near stall point, there are three critical regions in the passage, labeled I-III. Among them, the distribution of driving force in region I shows most notable effect and agrees with the variation of

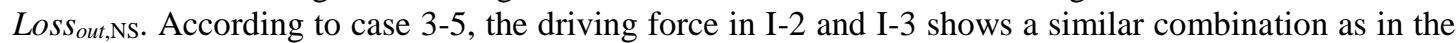
design point cases (case 2 and 3). However, since the corner separation develops from more upstream region for near stall point, the direction of the driving force in region I-1 should be of most significance. All the cases with lower Loss $_{\text {out }, \mathrm{NS}}$, show a driving force against the SS corner in I-1 while the alternative cases show an opposite direction. It is known that the accumulation of low energy fluid in the corner separation can be relieved by the former. Therefore, a driving force against the secondary flow in I-1 is believed to be essential for loss reduction near stall point; otherwise, the corner separation will be intensified and induce higher total pressure loss.

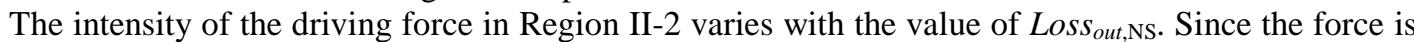
in the same direction even for cases with higher loss generation (case 1 and 2), it is deduced that this force is only beneficial rather than critical for loss reduction. The driving force in Region III does not show obvious negative or positive effect on the loss reduction.

Therefore, an effective flow control method for the near stall point should weaken the hub cross flow at the very front region on the SS-half of the passage. It can be accomplished by a reverse driving force against the hub secondary flow in the outer region of the front SS corner. At the same time, a stronger driving force near the mid-chord SS corner for acceleration of the cross flow is also helpful.

Compared with the result at design point, the corner separation is also influenced by the driving force around the front SS but the critical region of flow control is located in a different area. This explains why loss reduction at design and near stall point are generally independent with each other, as indicated by the Loss $_{\text {out,DP }}$ - Loss $s_{\text {out,NS }}$ projection in Fig.15, yet not completely independent. This finding is indicative of the possibility of using a profiled end wall to reach higher efficiency as well as better stability in axial compressor stages.

\section{Conclusion}

This paper researched a highly-loaded compressor cascade to determine the effective end wall profiling rules for compressors. A correlation analysis was applied for over $600 \mathrm{PEW}$ cases to find out the general principles for PEW design by clarifying how the variation of loss, secondary flow and passage vortex intensity interact with each other under the influence of profiled end wall. It was shown that

i. the control of corner separation is synchronous with that of span-wise blockage, and is of most importance for reducing total pressure loss over the full operating range.

ii. At design point, the effect of PEW would is most apparent in the rear part of passage. The variation of overall loss shows an approximately inverse relation with secondary flow intensity and is almost independent of the passage vortex. Therefore it is not infeasible to reduce loss at the design point by limiting the development of passage vortex or globally reducing the secondary.

iii. At the near stall point, the influence of PEW shows equal influence on the front and rear part of passage. The variation of overall loss, the intensity of overall secondary flow and the strength of the passage vortex almost change in the same direction because of the wide spreading corner separation. It is thus inferred that when significant corner separation exists in the passage, a 
reduction of loss can be accomplished by limiting the development of the passage vortex or suppressing the overall secondary flow.

A further optimization was carried out to find out the most effective way of implementing non-axisymmetric end wall profiling. To make the results practically applicable, profiling rules are were proposed focusing on the control of secondary flow by an analysis based on the "driving force" of PEW. It was found that:

iv. To reduce total pressure loss at the design point, the critical profiling region was located mainly at the SS-rear half of passage. PEW should induce a wide range of reverse driving force near the pitchwise center location, a local enhancing force near the SS and a narrow strip of reverse force alongside the SS corner to weaken the accumulation of low energy fluid from PS to SS in a wide streamwise range. This would also promote a locally stronger climb flow to reduce total pressure loss. Otherwise, an acceleration of cross flow near the front SS corner would intensify the corner separation and increase total pressure loss.

v. As for profiling at the near stall point, the critical region was located near the front SS corner. A driving force against the hub secondary flow in this region was essential for loss reduction. Otherwise, the corner separation would become more significant and increase the total pressure loss. Besides, the intensity of driving force near the mid-chord SS corner was also beneficial for loss reduction.

vi. The critical regions for loss reduction at design and near stall points have different locations in the passage. Therefore, it can be inferred that the loss control of different operating points is generally independent and not conflicting.

\section{Acknowledgement}

The work is funded by National Natural Science Foundation of China (Grant No. 51576162 and 51236006) and Doctorate Foundation of Northwestern Polytechnical University (CX201519).

\section{Reference}

1. Lei, V. M., Spakovszky, Z. S. and Greitzer E. M. A Criterion for Axial Compressor Hub-Corner Stall. Journal of Turbomachinery, 2008,Vol.130. DOI: 10.1115/1.2775492.

2. Rose, M. G. Non-Axisymmetric endwall profiling in the HP NGV's of an Axial Flow Gas Turbine, ASME paper, 94-GT-249.

3. Hu, S. Z., Lu, X. G., Zhang, H. W. and Zhu, J. Q. Numerical Investigation of a High-subsonic Axial-flow Compressor Rotor with Non-axisymmetric Hub Endwall, Journal of Thermal Science,2010, Vol.19, pp.14-20.DOI: 10.1007/s11630-010-0014-8.

4. Wu, J. C., Lu, X. G. and Zhu, J. Q. Secondary flow analysis for non-axisymmetric endwall on the highly-loaded compressor cascade, Journal of Aerospace Power,2011, Vol. 26, pp. 1362-1369.

5. Liu, B., Cao, Z. Y., and Huang, J. Design Optimization for a Transonic Compressor with Implementation of Non-axisymmetric Endwall Contouring, Journal of Propulsion Technology, 2012,Vol. 33, pp. 689-694.

6. Zhao, W. G., Jin D. H. and Gui, X. M. Design Optimization of Non-axisymmetric Endwall Contouring in Compressor Cascade, Journal of Engineering Thermophysics, 2013, Vol. 34, pp.1047-1050.

7. Reutter, O., Stefan H. P., Hergt, A. and Nicke. E. End wall Contouring and Fillet Design for Reduceing Losses and Homogenizing the Outflow of a Compressor Cascade, ASME paper, GT2014-25277, DOI: 10.1115/ GT2014-25277.

8. Harvey, N. W. Some Effects of Non-Axisymmetric End wall Profiling on Axial Flow Compressor Aerodynamics. Part I: Linear Cascade Investigation. ASME paper, GT2008-50990. DOI: 10.1115/GT2008-50990.

9. Harvey, N. W. and Offord, T. P. Some Effects of Non-Axisymmetric End wall Profiling on Axial Flow Compressor Aerodynamics. Part II: Multi-Stage HPC CFD Study. ASME paper, GT2008-50991, DOI: 10.1115/ GT2008-50991.

10. Reising, S. and Schiffer, H. Non-Axisymmetric End wall Profiling in Transonic Compressors. Part I: Improving the Static Pressure Recovery at Off-Design Conditions by Sequential Hub and Shroud End wall Profiling. ASME paper, GT2011-59133, DOI: 10.1115/ GT2011-59133.

11. Reising, S and Schiffer, H., Non-Axisymmetric End wall Profiling in Transonic Compressors. Part II: Design Study of a Transonic Compressor Rotor Using Non-Axisymmetric End walls Optimization Strategies and Performance. ASME paper, GT2011-59134, DOIi: 10.1115/ GT2011-59134.

12. Lepot, I., Mengistu, T. and Hiernaux S. Highly Loaded LPC Blade and Non Axisymmetric Hub Profiling Optimization for Enhanced Efficiency and Stability. ASME paper, GT2011-46261, DOI: 10.1115/ GT2011-46261. 
13. Li, X. J., Chu, W. L. and Wu Y.H. Numerical Investigation of Inlet Boundary Layer Skew in Axial-Flow Compressor Cascade and the Corresponding Non-Axisymmetric End wall Profiling, Proceedings of the Institution of Mechanical Engineers, Part A: Journal of Power and Energy, 2014, vol.228, DOI: 10.1177/0957650914532264.

14. Mahesh K. Varpe and A. M. Pradeep. Non-axisymmetric Endwall contouring in a Compressor Cascade with Tip Gap, ASME paper, GT2014-26725, DOI: 10.1115/ GT2014-26725.

15.Zhang, Y. F. Investigations of End wall flow behavior and its control strategies in Highly-loadeded Compressor," PhD Thesis, Northwestern Polytechnical University, P.R. China, 2010.

16. Zhang, Y. F., Mahallati, A. and Benner, M. Experimental and Numerical Investigation of Corner Stall in a Highly-Loaded Compressor Cascade. ASME paper, GT2014-27204, DOI: 10.1115/ GT2014-27204.

17. Schobeiri, M. T. and Lu, K. End wall Contouring Using Continuous Diffusion: A New Method and its Application to a Three-Stage High Pressure Turbine. Journal of Turbomachinery, 2014, Vol.136, DOI: $10.1115 / 1.4023970$.

\section{Appendix}

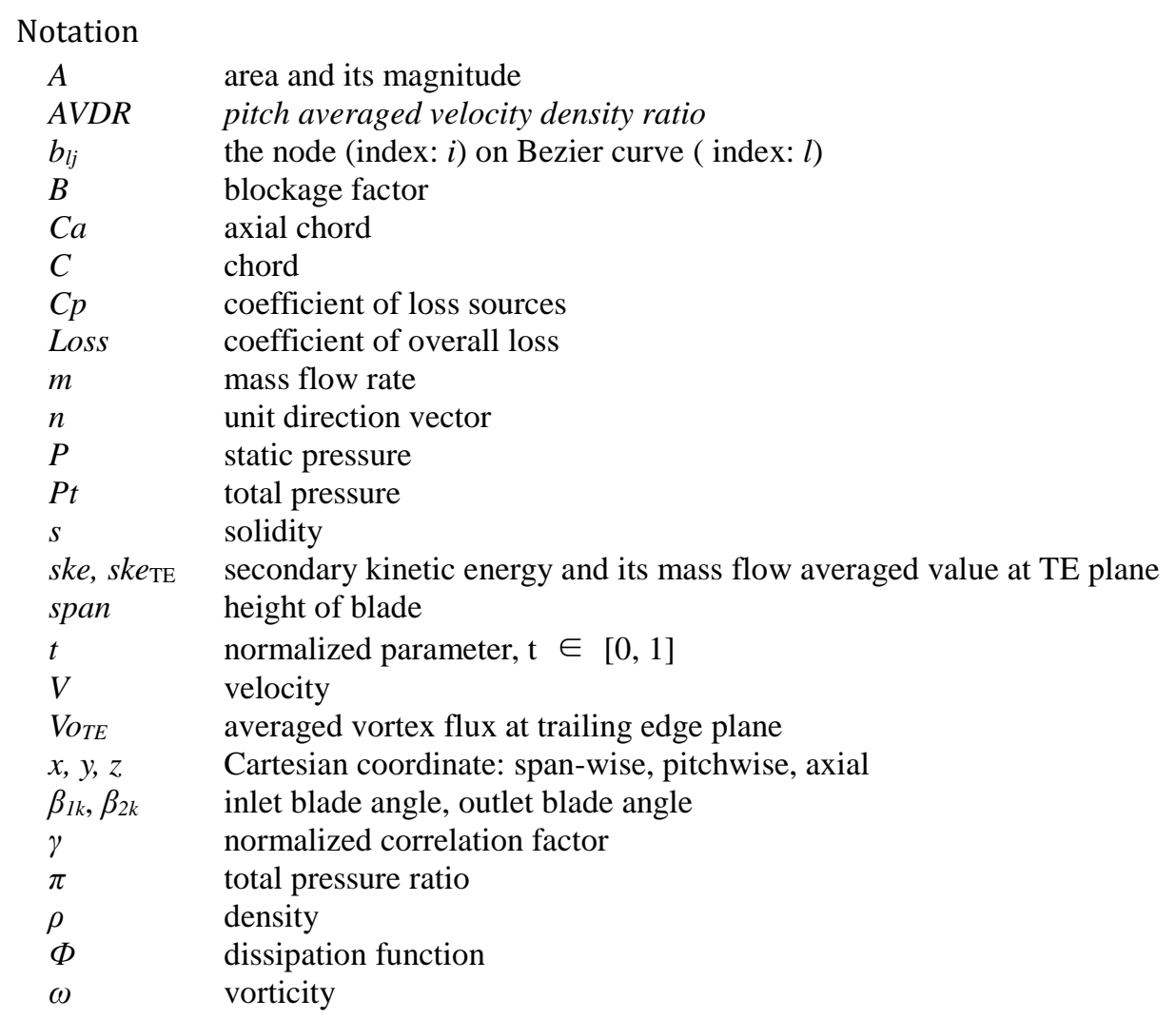

